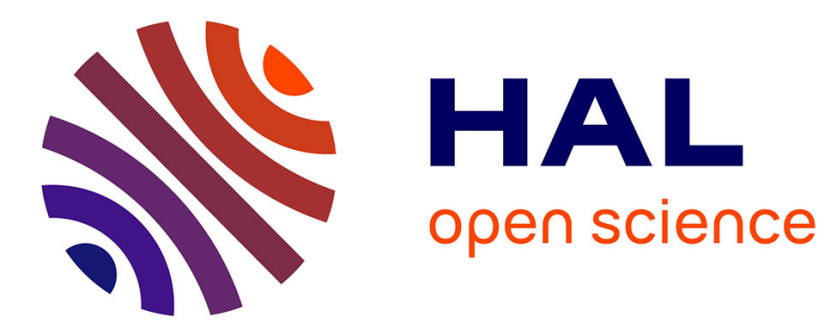

\title{
Mixed dimensional modeling of reinforced structures
}

Benoît Lé, Grégory Legrain, Nicolas Moes

\section{To cite this version:}

Benoît Lé, Grégory Legrain, Nicolas Moes. Mixed dimensional modeling of reinforced structures. Finite Elements in Analysis and Design, 2017, 128, pp.1 - 18. 10.1016/j.finel.2017.01.002 . hal01432692

\section{HAL Id: hal-01432692 \\ https://hal.science/hal-01432692}

Submitted on 24 Jan 2017

HAL is a multi-disciplinary open access archive for the deposit and dissemination of scientific research documents, whether they are published or not. The documents may come from teaching and research institutions in France or abroad, or from public or private research centers.
L'archive ouverte pluridisciplinaire HAL, est destinée au dépôt et à la diffusion de documents scientifiques de niveau recherche, publiés ou non, émanant des établissements d'enseignement et de recherche français ou étrangers, des laboratoires publics ou privés.

\section{(이) $\$$}

Distributed under a Creative Commons Attribution - NonCommercial - NoDerivatives| 4.0 


\section{Gem \\ Institut de Recherche en}

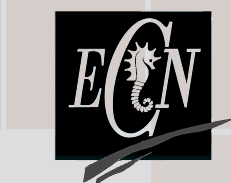

Centrale

Nantes

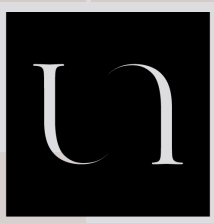

UNIVERSITÉ DE NANTES

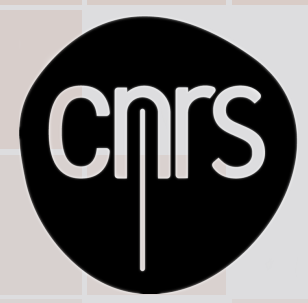

\section{Mixed dimensional modeling of reinforced structures}

Benoît Lé, Grégory Legrain and Nicolas Moës

\section{GeM Institute}

GeM Institute UMR CNRS 6183

Université Bretagne Loire

École Centrale de Nantes / Université de Nantes / CNRS,

1 Rue de la Noë, BP92101, 44321 Nantes, France.

Preprint submitted to: Finite Element Analysis and Design 


\title{
Mixed dimensional modeling of reinforced structures
}

\author{
Benoît Lé, Grégory Legrain and Nicolas Moës
}

GeM, UMR CNRS 6183, École Centrale de Nantes, 1 rue de la Noë 44321, Nantes, France

\begin{abstract}
SUMMARY
Using thin reinforcements is a common way to strengthen structures, as in reinforced concrete for example. From a numerical point of view, dealing with these reinforcements is tedious, because of their diameter which is usually small compared to the characteristic dimensions of the structures, therefore requiring very fine meshes to represent them accurately. In this paper, a new approach allowing to mix a volumic and a lineic modeling of the reinforcements is proposed. Fine meshes with a volumic representation of the reinforcements are used in the zones of interest of the structure, whereas coarser meshes associated to lineic elements are used in the rest of the structure in order to decrease the computing times. A methodology to ensure the transition between both modeling is proposed, so that the results in the zones of interest are similar to the results that would be obtained with a full volumic representation of the reinforcements. The efficiency of the method is illustrated on several examples, involving linear elasticity and plasticity.
\end{abstract}

\section{Preprint submitted to: Finite Element Analysis and Design}

KEY WORDS: Reinforced structures, Reinforcements, Extended Finite Element Method, Bar elements, Mixed dimensional modeling 


\section{Introduction}

Including "almost-1D" reinforcements, that is to say elements with one dimension much greater than the two others, is a common way to strengthen structures. A well-known example is reinforced concrete structures, where steel reinforcements are used in the zones where tensile stresses prevail, to make up for the low tensile strength of concrete. Numerical simulation of such structures is quite challenging from a geometrical point of view. Indeed, the diameter of the reinforcements is usually small compared to the characteristic dimensions of the structures (at least one order of magnitude smaller in the example of reinforced concrete structures), requiring very fine finite element meshes to represent them accurately. As a result, the necessary CPU resources and computation times can become very high.

In order to take into account these reinforcements with reasonable computing costs, different approaches were developed in the literature. The smeared model [1] consists in adding the stiffness of the reinforcements to the volumic elements containing them, introducing orthotropy in the reinforcements direction. This approach is well suited for structures were the reinforcements are perfectly bonded and are arranged in a regular pattern. More recently, David [2] developed a membrane model for regularly spaced reinforcements, based on asymptotic expansions, which is more accurate and allows to take into account loss of bond. In the discrete model, 1D bar elements are added along the edges of the volumic elements. It is more flexible than the smeared approach, since the layout of the reinforcements does not need to be regular anymore, but their paths still need to follow the nodes of the volumic mesh. Finally, the embedded approach $[3,4]$ allows any reinforcements layout, independently of the volumic mesh.

These approaches give good global results, but are not designed to get accurate local results around the reinforcements, as shown in [5] for instance. To sum up, there are two possibilities to model the reinforcements: using a 3D volumic mesh, which would give the most accurate results but is incompatible with industrial studies because of the too large computing costs, or using a smeared or a 1D representation of the reinforcements, decreasing the computing costs but leading to inaccurate local results. The approach proposed in this paper rests upon the idea that, when performing the finite element analysis of any structure, it is often possible to identify zones of interest, that is to say, parts of the structures where accurate results are wanted (because of stress concentrations, or because these are critical parts for which one wants to know how it will deteriorate, etc). Usually, fine meshes are used in such zones, whereas coarser meshes are used in the rest of the structure to decrease computing costs. Now, applying this idea to reinforced structures, we propose in this paper a method that allows to use a volumic representation of the reinforcements in the zone of interest, and a $1 \mathrm{D}$ representation in the rest of the structure. The volumic representation in the zone of interest will allow to get results as accurate as possible, especially close to the reinforcements, whereas the $1 \mathrm{D}$ representation will allow to use coarser meshes away from the zone of interest, therefore reducing computing times. The transition between both representations will ensure that 
the results in the fine zone will be close to the results that would have been obtained with a full volumic representation of the reinforcements in the whole structure.

Combining $1 \mathrm{D} / 2 \mathrm{D}$ and $3 \mathrm{D}$ models in the same computation has already been done, as in $[6,7]$ where coupling $1 \mathrm{D}$ beams or $2 \mathrm{D}$ plates to $3 \mathrm{D}$ bodies was achieved by equating the work at the interface between both representation, resulting in constraint equations between $1 \mathrm{D} / 2 \mathrm{D}$ and 3D degrees of freedom. In [8], Nitsche's method [9] is applied to the beam/solid and plate/solid coupling. However, the main difficulty with the existing methods is the need to ensure the compatibility between the beam/plate particular kinematic and the volumic one. In this paper, we will consider structures where the reinforcements have a small enough diameter so that we can assume that they behave like bar element, working in tension/compression. However the proposed approach would still be useful if the bending energy was taken into account [10].

This paper is organized as follow: sections 2 and 3 introduce the numerical tools involved in the proposed method, as well as their limitations. In section 4 , the solution to combine these tools in order to answer the issues raised above is explained. Some results to illustrate this method are shown in section 5. Finally conclusions are drawn in section 6 .

\section{Volumic model}

The most direct approach to model reinforcements would be to mesh them using volumic finite elements. This process may be complicated, considering their small diameter, their number, and their path which may be complicated (intersecting or tangent reinforcements, curved paths, etc). However, the eXtended Finite Element Method (X-FEM) can be used, as done in the work of [11]. This method was first introduced by Moës et al. [12] to deal with crack propagation, with meshes independent of the crack path. Based on the concept of Partition of Unity [13], it relies on the use of enrichment functions to introduce discontinuities into the classical finite elements, as well as level-set functions to locate these discontinuities. The enrichment strategy proposed in [14], dedicated to the analysis of inclusions, is used in this paper for the volumic part of the reinforcements, and will be reminded below.

\subsection{The eXtended Finite Element Method (X-FEM)}

Consider the bidimensional problem depicted in figure 1 a., a circular inclusion made of a material $\mathrm{A}$, in a rectangular plate made of a material $\mathrm{B}$. The plate is meshed with elements whose nodes do not coincide with the material interface. The set $I$ denotes the nodes of the mesh, whereas $J$ is the set of the nodes which need to be enriched. $J$ is defined as the set of nodes belonging to the elements crossed by the material interface (cf. figure $1 \mathrm{a}$ ). The X-FEM approximation consists in finding a displacement solution of the form: 


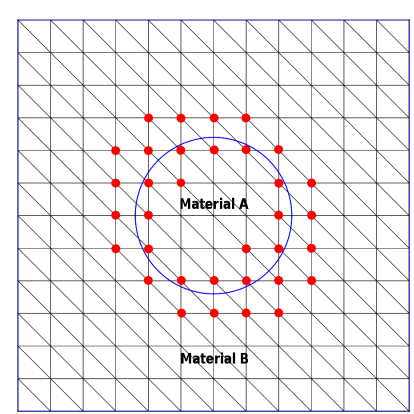

a.

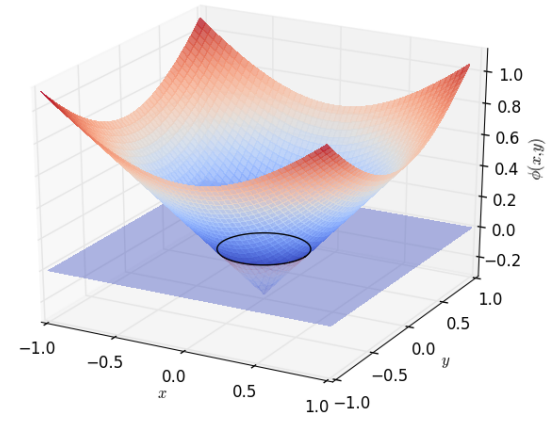

b.

Figure 1: Representation of a circular inclusion in a square plate, using the X-FEM method. a. Definition of the enriched nodes. b. Localization of the interface using a level-set function

$$
\mathbf{u}^{h}(\mathbf{x})=\sum_{i \in I} N_{i}(\mathbf{x}) \mathbf{u}_{i}+\sum_{j \in J} N_{j}(\mathbf{x}) F(\mathbf{x}) \mathbf{a}_{j} \quad \mathbf{u}_{i}, \mathbf{a}_{j} \in \mathbb{R}^{2}
$$

Where $\left(N_{k}\right)_{k=i, j}$ are the classical finite element approximation functions of node $k, \mathbf{u}_{i}$ are the classical finite element degrees of freedom, $\mathbf{a}_{i}$ are the enriched degrees of freedom and $F$ is the enrichment function. In this paper we consider that the two materials are perfectly bonded: therefore, $\mathbf{u}^{h}$ must be continuous over the domain. However, because of the change of material properties, the strain will be discontinuous at the interface. The enrichment function $F$ is thus chosen continuous, but with a discontinuous derivative.

To define $F$, the position of the interface must be determined. A level-set function $\phi$ is introduced to locate the interface $\Gamma$ between material $\mathrm{A}$ and $\mathrm{B}$ so that:

$$
\Gamma=\left\{\mathbf{x} \in \mathbb{R}^{2}: \phi(\mathbf{x})=0\right\}
$$

$\phi(\mathbf{x})$ is chosen to be positive if $\mathbf{x}$ is outside $\Gamma$, negative if $\mathbf{x}$ is inside $\Gamma$ and equals zero if $\mathbf{x}$ is on $\Gamma$. An example of level-set defining the inclusion of figure $1 \mathrm{a}$. is given in figure $1 \mathrm{~b}$. The main example of level-set function is the signed distance function to the interface:

$$
\phi(\mathbf{x})= \pm \min _{\mathbf{x}_{\Gamma} \in \Gamma}\left\|\mathbf{x}-\mathbf{x}_{\Gamma}\right\|
$$

In the particular case of a cylindrical reinforcement, we use the following level-set function:

$$
\phi(\mathbf{x})=\min _{\mathbf{x}_{\Lambda} \in \Lambda}\left\|\mathbf{x}-\mathbf{x}_{\Lambda}\right\|-r
$$




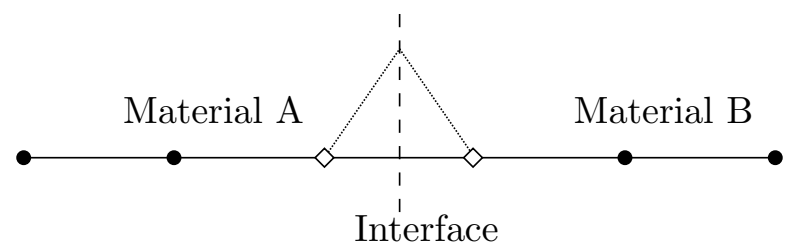

Figure 2: Ridge enrichment in 1D. The enriched nodes are the diamond nodes.

where $r$ is the radius of the reinforcement and $\Lambda$ is its center-line (the $1 \mathrm{D}$ curve defining the reinforcement path through its center). From a numerical point of view, $\phi$ is discretized using the linear finite element shape functions $N_{i}$ :

$$
\phi(\mathbf{x})=\sum_{i \in I} N_{i}(\mathbf{x}) \phi_{i}
$$

where the $\phi_{i}$ are the nodal values of the level-set function. Now that the position of the interface is known, the enrichment function can be defined. We consider the ridge function from [14]:

$$
F(\mathbf{x})=\sum_{i \in I} N_{i}(\mathbf{x})\left|\phi_{i}\right|-\left|\sum_{i \in I} N_{i}(\mathbf{x}) \phi_{i}\right|
$$

$F$ is shown in $1 \mathrm{D}$ on figure 2 .

Some authors also proposed to treat material interfaces at the integration point level [15]. However, as illustrated in appendix 7, this approach has a lower convergence rate than the X-FEM. On the contrary, for a given mesh density, the X-FEM and conforming FEM lead to similar results without meshing burden, which motivates the use of the XFEM here.

\subsection{Numerical aspects}

A first remark about the use of level-sets to implicitly represent interfaces is the fact that they are linearly approximated (cf. figure 3 ) ; the value of $\phi_{i}$ is known at each node $i$ of the mesh. If node $i$ is inside $\Gamma, \phi_{i}<0$, if node $i$ is outside $\Gamma, \phi_{i}>0$. The intersection of $\Gamma$ with the edges of mesh can be easily computed using the linear interpolation on the triangular element (cf. equation (5)). This implies that the quality of the geometries discretized using level-set functions will depend on their curvature (the less the curvature, the better the approximation).

The second remark is about numerical integration. Since the derivative of $\mathbf{u}^{h}$ is now discontinuous inside the elements crossed by $\Gamma$, classical Gauss integration rules are not accurate anymore. The elements which are crossed by $\Gamma$ are divided into conforming subcells (cf. figure 3), then Gauss integration is performed using the Gauss integration 


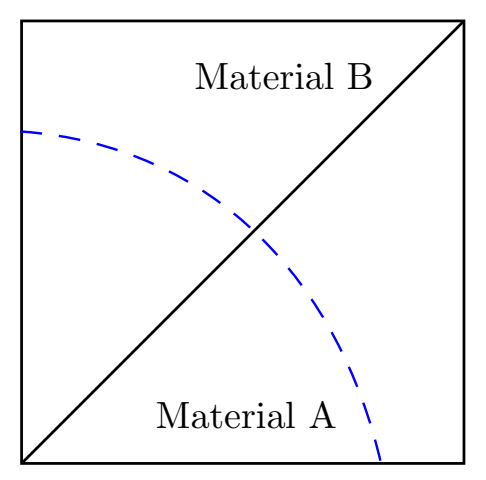

a.

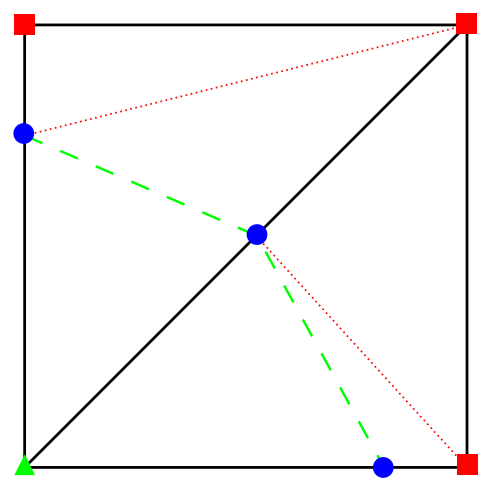

b.

Figure 3: Representation of an interface over a mesh using level-set functions. Black thick lines: mesh edges. a. Blue dashed line: real interface b. Green triangle nodes: $\phi(\mathbf{x})<0$. Red square nodes: $\phi(\mathbf{x})>0$. Blue dots: $\phi(\mathbf{x})=0$. Green dashed line: approximate geometry represented with the level-set. Each triangle appearing in $\mathrm{b}$. will be used for the numerical integration

points of each subcell. Note that subcells do not bring any new degrees of freedom. The only purpose is to reach a proper level of accuracy for the integration with a very limited number of integration points.

\subsection{Limitations of the 3D approach}

Now we consider the 2D case of a rectangular plate, with a unique reinforcement (cf. figure 4). Considering that it is perfectly linked to the plate material, it can be assimilated to an inclusion and modeled using the X-FEM method, as presented above. The level-set function is the one defined by equation (4). Following the idea presented in the introduction, two meshes are considered: a fine mesh, and a second one where the right part was derefined (assuming that it is out of the zone of interest). The approximated geometry obtained by the level-set is given for both meshes in figure 4 . With the fine mesh, the reinforcement is correctly represented (in 2D it is a flatten rectangle with width $2 r$ ). On the other hand, the reinforcement approximated on the second mesh shows strong geometrical errors, almost disappearing where the mesh is really coarse. The corresponding finite element computation will obviously give wrong results, both locally and globally.

These matter islands are due to the nodes whose support (set of elements connected to a node) is cut twice by $\Gamma$, as illustrated in figure 5 . The support of $\mathrm{A}$ is cut twice by $\Gamma$. Let us have a closer look at the intersection between $\Gamma$ and edges $\mathrm{AB}$ and $\mathrm{AC}$ of the mesh. Because of equation (4), the value of $\phi_{A}$ is equal to the distance from A to the lower part of $\Gamma$. The intersection between $\Gamma$ and $\mathrm{AB}$ is correctly computed, but because the value of $\phi_{A}$ underestimates the distance from $\mathrm{A}$ to the upper part of $\Gamma$, the intersection 

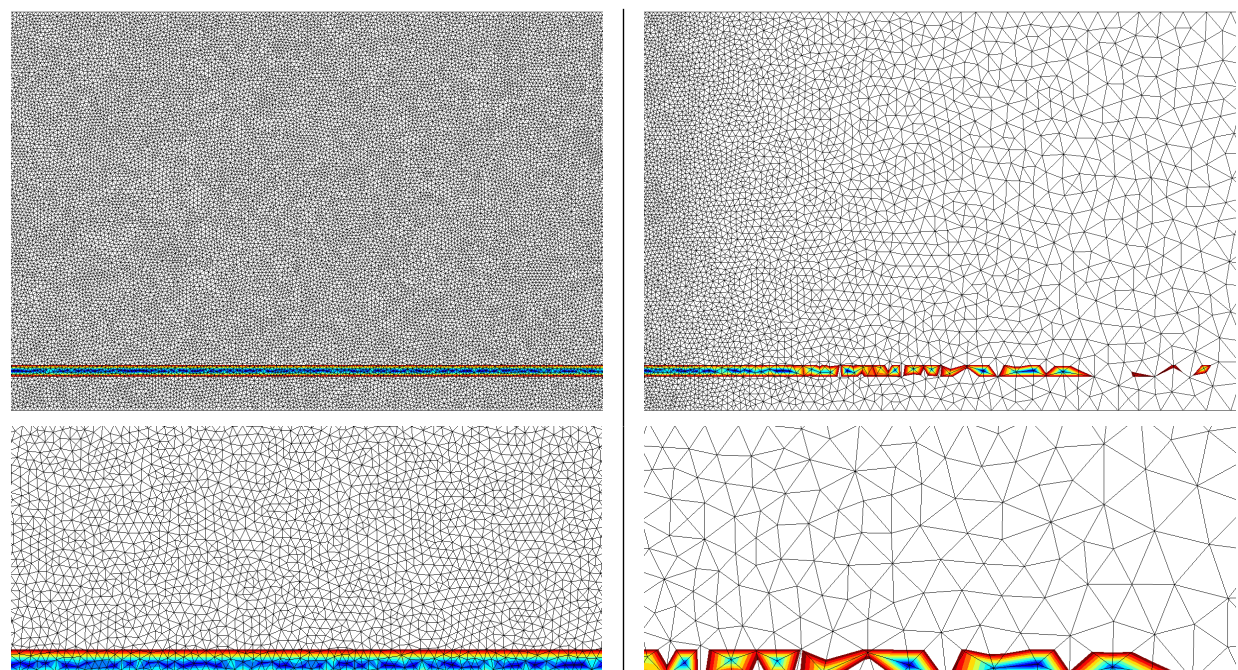

(1)
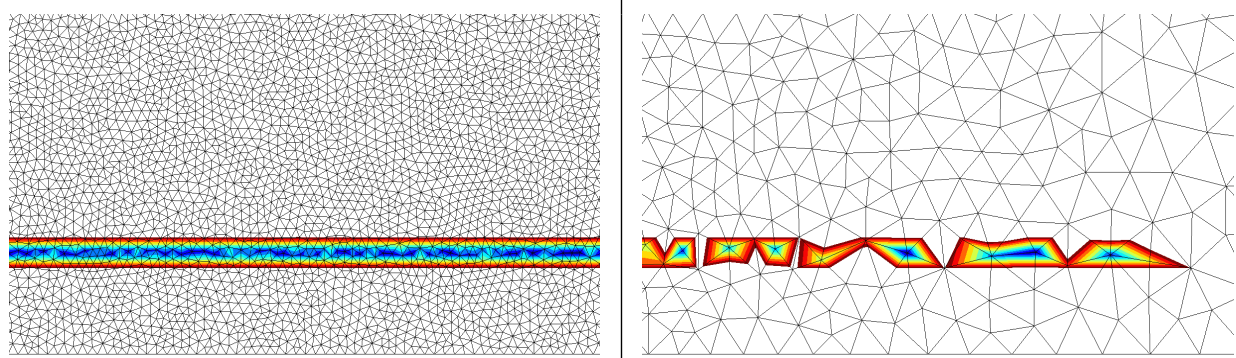

Level-set

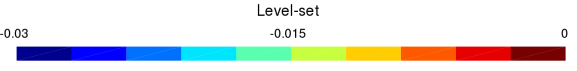

Level-se

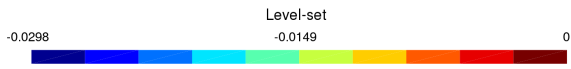

Figure 4: Using level-set to represent reinforcements. Left: with a fine mesh in the whole structure. Right: with a coarse mesh in part of the structure. Top: overview. Bottom: zoom in the transition between the fine and the coarse mesh. 


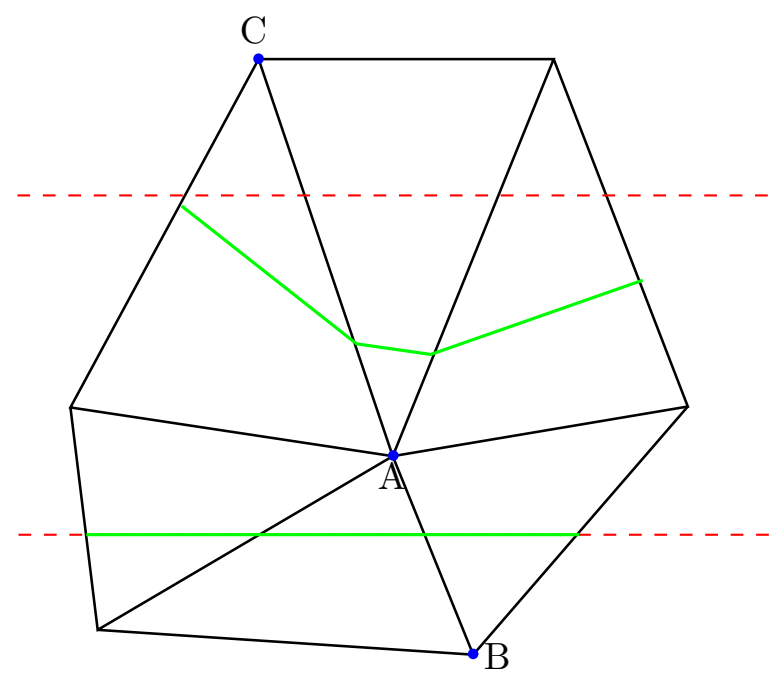

Figure 5: Zoom on a node with a support cut twice by $\Gamma$. Red dashed lines: real geometry of $\Gamma$. Full green lines: interpolated geometry of $\Gamma$

between $\Gamma$ and $\mathrm{AC}$ is closer to $\mathrm{A}$ than it should be. It is important to emphasize that this definition ensure that if there are some errors in the approximation of $\Gamma$, it will always result in local underestimation of the volume of the reinforcement (some "matter" can be removed, but not added). Finally, the elements where the reinforcement seems to disappear are due do the fact that there is no node inside $\Gamma$, therefore the sign of $\phi$ does not change, making it unable to locate the inner part of the reinforcement.

\section{Linking truss and 3D model}

Another way to model reinforcements without using a costly $3 \mathrm{D}$ representation is to use bar elements. The reinforcements are meshed with lineic element, whose nodes are not necessarily matching the nodes of the volumic mesh, cf. figure 6 . A reinforcement node $a$ is inside a cubic volumic element whose nodes are called $\left(b_{i}\right)_{i=1, \ldots, 8}$. The position of $a$ inside the volumic element is $\mathbf{x}_{a}$. Considering perfect bond between both materials, the reinforcement degrees of freedom are linked to the volumic degrees of freedom according to equation (7):

$$
\mathbf{u}_{a}=\sum_{i=1}^{8} N_{i}\left(\mathbf{x}_{a}\right) \mathbf{u}_{b i}
$$

In section 4.1 we will see that bar elements will be used with X-FEM in the same computation. Therefore, in the case where a bar node is located inside an enriched volumic element, (7) must be modified in order to take into account the X-FEM enrichment (1): 


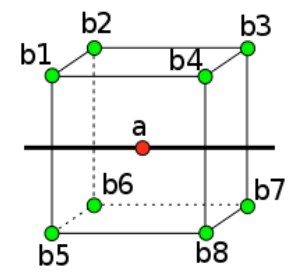

Figure 6: Reinforcement node inside a volumic element

$$
\mathbf{u}_{a}=\sum_{i=1}^{8}\left(N_{i}\left(\mathbf{x}_{a}\right) \mathbf{u}_{b i}+N_{i}\left(\mathbf{x}_{a}\right) F\left(\mathbf{x}_{a}\right) \mathbf{a}_{b i}\right)
$$

The bar degrees of freedom are eliminated from the calculation through equation (7) (or equation (8) if bar elements are used with the X-FEM enrichment), which is somehow equivalent to considering that the stiffness of the bar elements is added to the stiffness of the surrounding volumic elements (green elements on figure $7 \mathrm{~b}$.). Besides allowing to use coarser meshes around the reinforcements, using bar elements simplifies the meshing procedure since the volumic and lineic meshes can be independent. This method allows to get good global results (force-displacement curve for instance), but is obviously not as accurate as a volumic representation of the reinforcements as shown by [5]. Indeed, two drawbacks can be underlined:

- The overall stiffness of the structure is overestimated. Let us consider the example of figure 7 a. A reinforcement, denoted $\Omega_{\text {inclusion, }}$, is embedded in a matrix called $\Omega_{\text {matrix }}$. The structure is meshed using coarse $2 \mathrm{D}$ elements, not taking into account the interface between $\Omega_{\text {inclusion }}$ and $\Omega_{\text {matrix }}$ (cf. figure $7 \mathrm{~b}$.). These $2 \mathrm{D}$ elements have the material properties of the matrix material, and the reinforcement is introduced with 1D bar elements having the material properties of the inclusion material. The discretized stiffness of the geometrical domain $\Omega_{\text {inclusion }}$ is the sum of two terms: obviously, the stiffness of the reinforcements coming from the 1D elements, and a second term which corresponds to the part of the 2D finite elements inside $\Omega_{\text {inclusion }}$, but have the material properties of the matrix (gray part in figure $7 \mathrm{~b}$.). Therefore the stiffness of $\Omega_{\text {inclusion }}$ is overestimated. However, the reinforcements are usually stiffer than the matrix and their diameter is small, so the influence of this extra stiffness should remain small in most of the cases.

- One can see from figure $7 \mathrm{~b}$. that if the size of the $2 \mathrm{D}$ elements around the bar elements becomes small, the stiffness of the reinforcements is distributed over a band of vanishing thickness, which may lead to non-physical stress concentrations (see section 5.2.2).

It is also possible to consider that the link between bar and volumic elements is not perfect, by allowing a slip between the bar nodes and the volumic ones [16], but in this paper we will consider only perfect bond. 


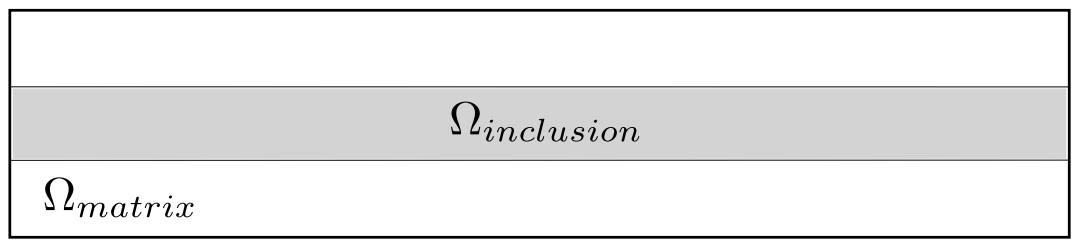

a.

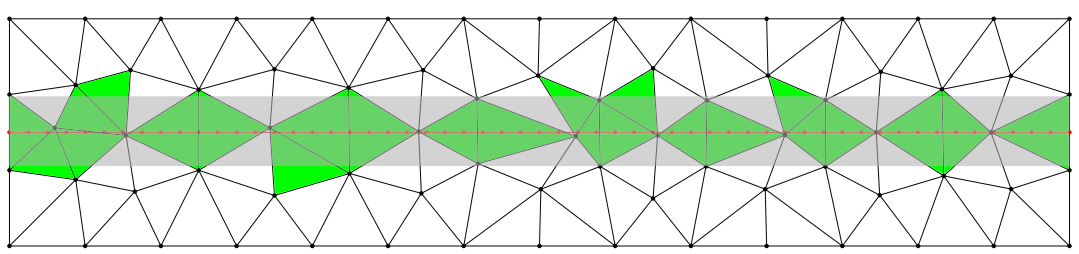

b.

Figure 7: Redistribution of the reinforcements stiffness in the volumic elements. a. Domains definition. b. Mesh of the domains, using 1D bar elements.

\section{Mixing lineic and volumic models}

\subsection{Correction scheme}

It was shown in section 2 that X-FEM can be used to obtain a volumic representation of the reinforcements with meshes which do not need to coincide with their boundaries. However, if the mesh is too coarse, some geometrical errors can occur. The solution proposed in this section is to keep the geometry of the reinforcements approximated by X-FEM, which may be accurate in the zone of interest and potentially inaccurate where the mesh is coarse, and to use bar elements as presented in section 3 at the same time. Let us consider the example depicted in figure 8. A reinforcement is modeled using both X-FEM and bar elements, placed along the reinforcement path. In some part of the structure, the mesh is coarse enough so that at least one node has its support cut twice by $\Gamma$. The volumic part approximated with X-FEM and the lineic part are both assembled, but the stiffness of each bar element is weighted by a coefficient $\alpha_{e}$. Considering that each bar element delimitates a segment of the reinforcement (red dashed line in figure $8)$; the real volume of this segment can be computed as:

$$
\Omega_{e}^{r e a l}=S l_{e}
$$

where $S$ is the section of the reinforcement an $l_{e}$ the length of the bar element. Then $\alpha_{e}$ is computed by:

$$
\alpha_{e}=1-\frac{\Omega_{e}^{\text {num }}}{\Omega_{e}^{\text {real }}}
$$


where $\Omega_{e}^{n u m}$ is the volume of the segment of the reinforcement that could be approximated using X-FEM (darken color part inside the red dashed line rectangle in figure 8). $\Omega_{e}^{\text {num }}$ is computed as follow:

1. The volumic element around each bar element are divided into subelements, using the level set $\phi$, according to section 2.2

2. The subelements close to the extremities of the segment are cut once again by two planes normal to the bar element at the bar nodes

3. $\Omega_{e}^{\text {num }}$ is then the sum of the volumes of all the subelements resulting from step 1 and 2 which are inside $\Omega_{e}^{r e a l}$. These elements can be found easily because the value of $\phi$ is smaller or equal to zero at each of their nodes.
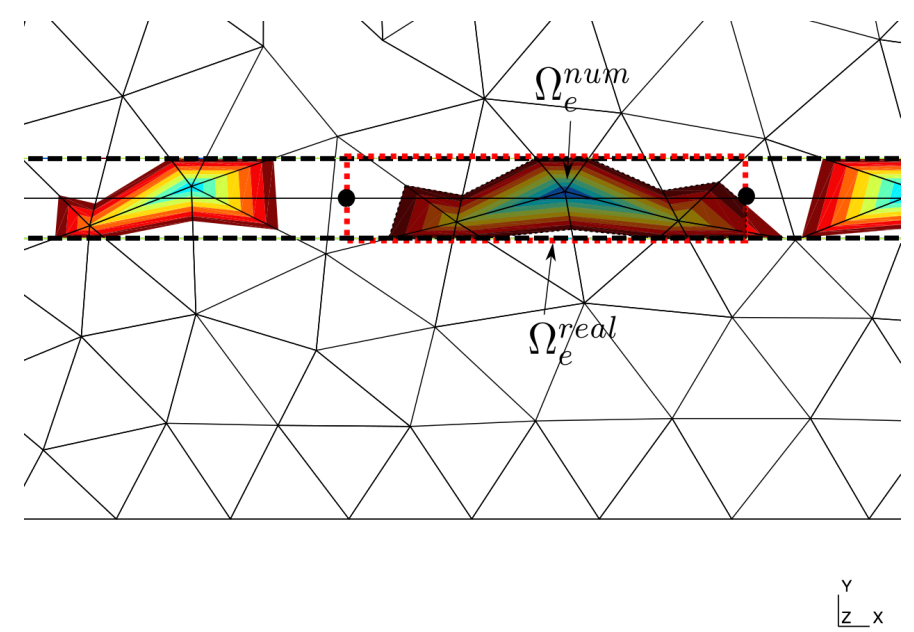

Figure 8: Computation of $\alpha_{e}$

To sum up, the bar elements can be seen as a correction to the X-FEM approximation, through the use of coefficient $\alpha_{e}$ computed by (10):

- $\alpha_{e}=0$ if $\Omega_{e}^{n u m}=\Omega_{e}^{\text {real}}$ : the segment of reinforcement is perfectly represented by $\mathrm{X}-\mathrm{FEM}$, the bar element is not taken into account, no correction is needed

- $\alpha_{e}=1$ if $\Omega_{e}^{\text {num }}=0$ : the mesh is very coarse, no node is inside the reinforcement, therefore it "disappears": a classical bar element is assembled

- $\left.\alpha_{e} \in\right] 0 ; 1\left[\right.$ if $0<\Omega_{e}^{\text {num }}<\Omega_{e}^{\text {real }}$ : the reinforcement is only partially represented by the level-set, the bar element brings the missing stiffness as a correction

Note that using these modified bar elements does not solve the local issues of X-FEM emphasized in section 2.3: in the coarse zone, the results around the reinforcements will 
still be inaccurate. However, from a global point of view, the stiffness of the structure in the coarse zone will be sufficiently correct so that the local results in the fine zone are almost identical to the results that would be obtained with a full volumic representation of the reinforcements in the whole structure. The extra stiffness around bar element $e$ mentioned in section 3 is now equal to $\alpha_{e} k_{e}^{\text {matrix }}$, where $k_{e}^{\text {matrix }}$ is the stiffness of $\Omega_{e}^{\text {real }}$ assuming that it is made only of the matrix material. Since $\alpha_{e}$ is smaller than 1 , the global stiffness of the structure should be better with the approach proposed in this section than with only $1 \mathrm{D}$ bar elements. This will be shown in numerical examples in section 5 .

\subsection{Flowchart}

Here is the general procedure to perform the finite element computation of a reinforced structure in linear elasticity with perfect bond, using the method presented in section 4.1:

1. Make a volumic mesh of the structure

2. Make a 1D mesh of the reinforcements

3. Compute the value of the level-set $\phi$ using equation (4). Note that the center-line of the reinforcement $\Lambda$ can be replaced by a $1 \mathrm{D}$ mesh. In this case the value of the level-set at each node of the volumic mesh is computed by a direct distance computation from this node to the 1D mesh, and by subtracting the radius of the reinforcement. Provided that enough bar nodes are used, the discretization error remains low, without influencing the computation times

4. Assemble the stiffness corresponding to the volumic part, according to the procedure given in section 2.2

5. For each bar element, compute coefficient $\alpha_{e}$

6. Assemble the stiffness corresponding to the $1 \mathrm{D}$ part of the reinforcements, multiplying the stiffness of each bar element by $\alpha_{e}$ and taking into account the relation (7)

7. Finally, solve the linear system 


\begin{tabular}{|l|l|l|}
\hline & Matrix & Reinforcements \\
\hline Young modulus $(\mathrm{Pa})$ & $40.10^{11}$ & $200.10^{11}$ \\
\hline Poisson ratio & 0.24 & 0.3 \\
\hline Yield stress (Pa) & $4.10^{6}$ & - \\
\hline $\begin{array}{l}\text { Hardening modulus } \\
(\mathrm{Pa})\end{array}$ & $-100^{6}$ & - \\
\hline
\end{tabular}

Table 1: Material properties

\section{Numerical examples}

The approach proposed in section 4 is applied to several examples. To illustrate the interest of the method, we consider structures with reinforcements stiffer than the surrounding matrix. Perfect bond is considered between both materials. In section 5.1 the matrix and the reinforcements are made of linear elastic materials. For the examples of section 5.2, non-linear test cases are considered. Softening models like damage mechanics would be a rather logical possibility as one of the main applications of the proposed method is reinforced concrete. However, these models need some regularization in order to avoid spurious mesh dependency [17], which is usually done by introducing non-locality in the problem. Taking this non-locality into account close to the reinforcements would require some developments which would be out of the scope of this paper. Therefore, an elastoplastic material with a simple isotropic hardening ( $J_{2}$-flow theory [18]) is considered for the matrix. This will not be representative of any particular kind of physical phenomenon, but will allow to get qualitative non-linear results. The hardening modulus is chosen negative (softening plasticity), to get a behaviour qualitatively similar to damage mechanics. The material properties of the matrix and the reinforcements are summarized in table 1 , and the behaviour at Gauss points for the matrix material is shown is figure 9 .

For each example, a zone of interest is identified. A mesh with elements fine enough to correctly represent the reinforcements is used in this zone. This mesh will be called the "computation mesh". The method presented in section 4 will be called the "mixed" method in what follows (mix between a volumic representation of the reinforcements with X-FEM and a 1D representation with bar elements). Unless otherwise specified, the obtained results are compared with two other methods:

- A computation performed with the same mesh, but using only truss elements in the whole structure. This method will be called the "bar" method.

- A computation performed with a fine mesh in the whole structure. The element size is the same as the the element size in the zone of interest of the computation mesh. This mesh will be called the "reference mesh". The reinforcements are represented using X-FEM. As the mesh is fine everywhere, the reinforcements are perfectly represented in $3 \mathrm{D}$, therefore these results will be taken as references. This 


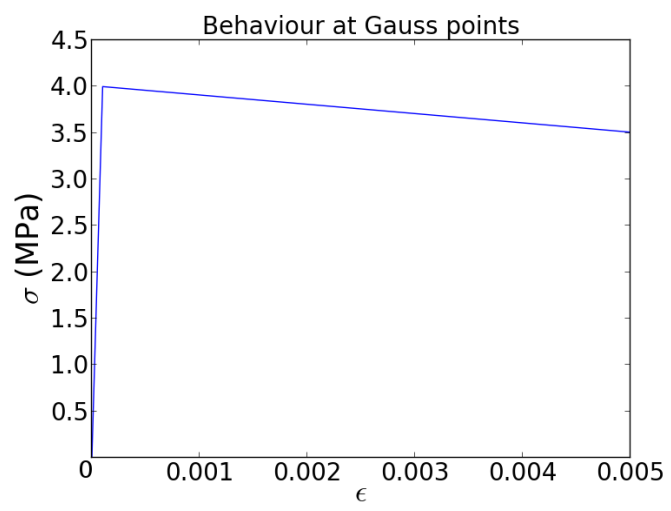

Figure 9: Behaviour at Gauss points of the matrix material

method will be called the "volumic" method.

All the meshes used in this section are made of linear elements.

\subsection{Elastic test cases}

\subsubsection{Three points bending 2D elastic}

The first example is a three points bending test in $2 \mathrm{D}$, under plane strain assumption. The geometrical characteristics of the test are given in figure 10: length $L=5.1 \mathrm{~m}$, height $h=0.8 \mathrm{~m}$, thickness $t=1 \mathrm{~m}$. The beam is strengthened with a reinforcement of radius $0.016 \mathrm{~m}$ in the upper part, and a reinforcement of radius $0.02 \mathrm{~m}$ in the lower part. The zone of interest is supposed to be at the center of the beam, and has a width $D$.

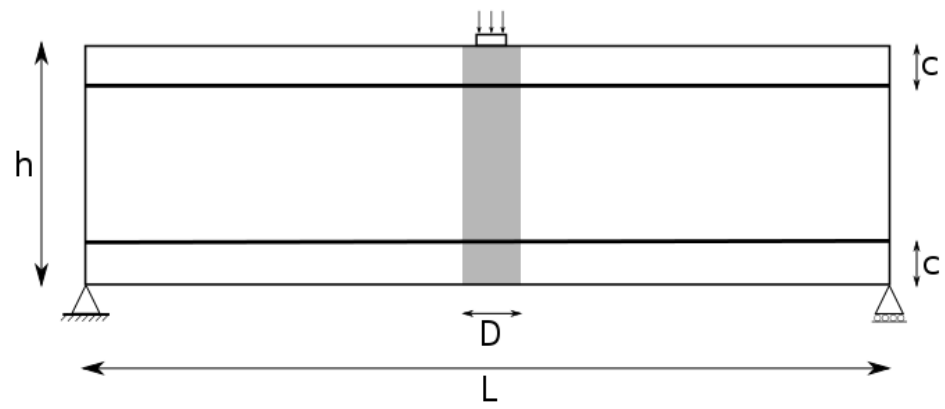

Figure 10: Geometry of the three points bending test and boundary conditions

For the computation mesh, the element size is $0.005 \mathrm{~m}$ in the zone of interest, and 0.05 $\mathrm{m}$ outside (cf. figure 11). For the reference mesh, the element size is $0.005 \mathrm{~m}$ in the whole 


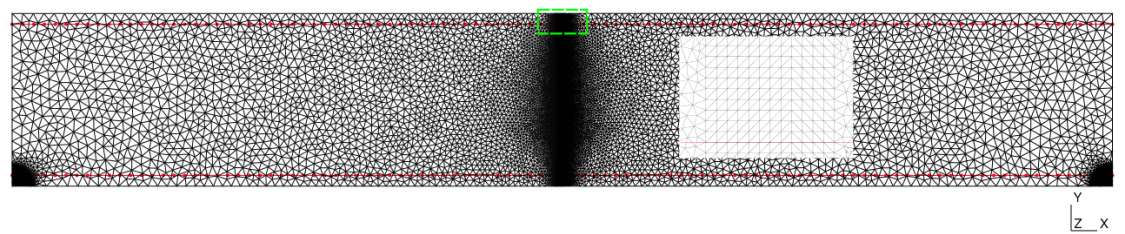

Figure 11: Mesh used for the three points bending test in linear elasticity. In the zone of interest, the elements are geometrically superimposed for the computation mesh and the reference mesh.

beam. The width of the zone of interest is $D=0.06 \mathrm{~m}$. Note that the element size does not seem really fine in the zone of interest compared to the reinforcements radius. Since in 2D, straight reinforcements are represented with flat rectangles, the mesh does not need to be really fine, which is not the case in $3 \mathrm{D}$ as illustrated in section 5.2.2. It just needs to ensure that no node has its support cut twice by the iso-zero of the level-set. The mesh in the zone of interest is made of squares cut into triangles (see figure 11). This is to ensure that the elements in this zone are exactly superimposed for the computation and the reference meshes, something which is necessary for the comparison between the different computations (cf. equation (14)). For this computation the mesh was also refined around the boundary conditions, to account for the singularities resulting from the imposition of a displacement on points.

The vertical displacement $v$ and the normal stress $\sigma$ along the upper reinforcement are plotted in figure 14, as well as the relative error between the mixed/bar method and the volumic method. For a 1D bar element, the computation of the normal stress is reminded:

$$
\sigma_{i j}=\frac{E}{l_{e}}\left(\boldsymbol{u}_{j}-\boldsymbol{u}_{\boldsymbol{i}}\right) \cdot \boldsymbol{v}
$$

where $\boldsymbol{u}_{\boldsymbol{i}}$ and $\boldsymbol{u}_{\boldsymbol{j}}$ are the displacement of the bar nodes. $\boldsymbol{v}$ the unitary vector orienting the bar element, going from node $i$ to node $j$. The same expression is used for the volumic and mixed methods (in the case of the volumic method, bar elements are used in the computation but their stiffness is null, they are used only to compute (11)). The relative error for the bar and the mixed method compared to the volumic method, are also computed :

$$
\eta_{v}^{b a r / m i x}=\frac{\left|v_{i}^{v o l}-v_{i}^{b a r / m i x}\right|}{\left|v_{i}^{v o l}\right|}
$$

for the displacement error, computed at each node $i$, where the subscripts $b a r / m i x / v o l$ refer to the bar, mixed and volumic method respectively, and 


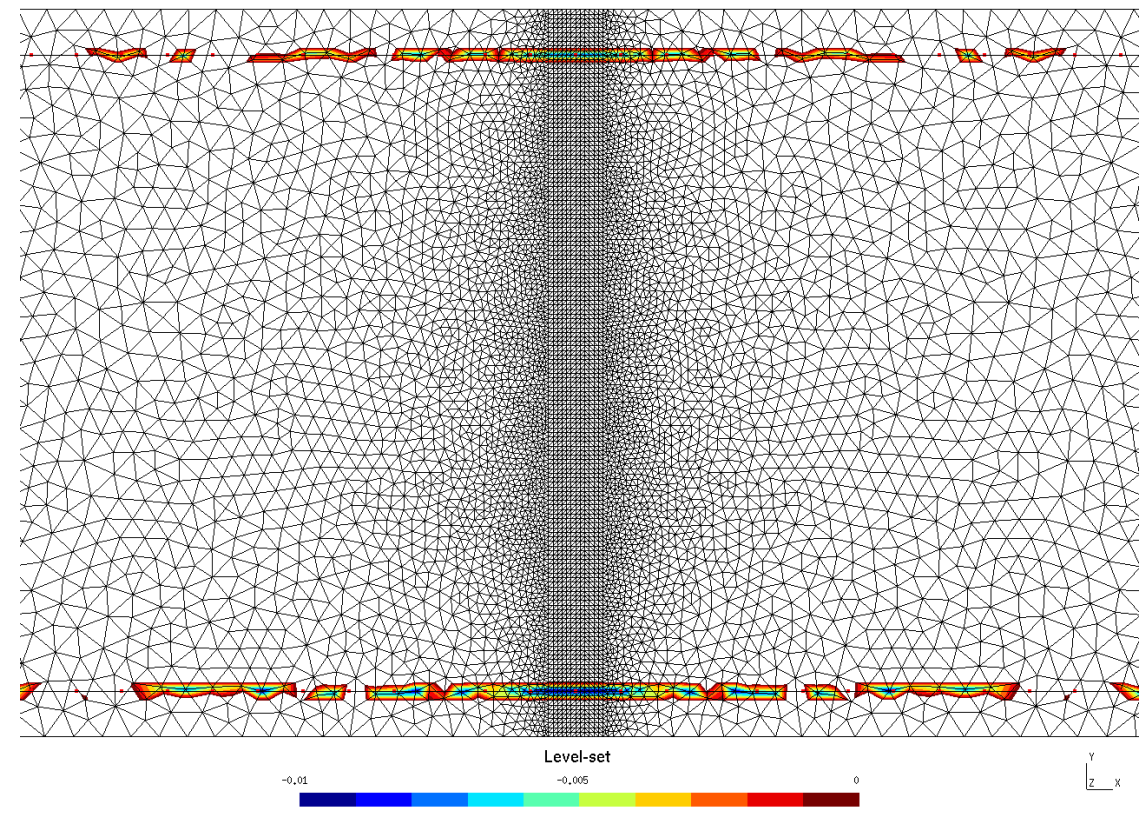

Figure 12: Approximated geometry of the reinforcements for the three points bending test (zoom on the center of the beam).

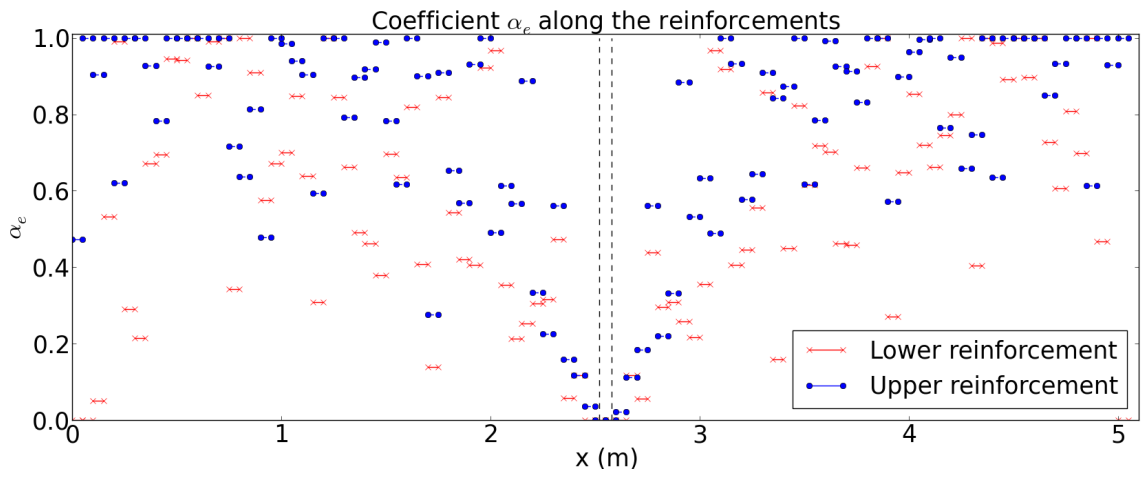

Figure 13: Coefficient $\alpha_{e}$ along the reinforcements for the three points bending test.

$$
\eta_{\sigma}^{b a r / m i x}=\frac{\left|\sigma_{i j}^{v o l}-\sigma_{i j}^{b a r / m i x}\right|}{\left|\sigma_{i j}^{v o l}\right|}
$$

for the stress error, computed at each bar element, defined by nodes $i$ and $j$.

The three methods give similar results. The effect of the extra-stiffness mentioned in section 3 can be observed, with a vertical displacement with the bar method smaller than the one obtained with the mixed method, which illustrates the fact that with the 
mixed method, this extra-stiffness is lower than with a pure bar method.. The error compared to the volumic method are lower with the mixed method than with the bar method, even if they are acceptable in both cases (less than 10\%). It can be noticed that in the fine zones around the boundary conditions, the bar method fails to capture the displacement and normal stress, which is not the case for the mixed method. The same conclusions can be drawn for the lower reinforcements.

To get local information about the mechanical field around the reinforcements in the zone of interest, the error for each element $e$ per unit volume is also computed:

$$
\eta_{e}^{b a r / m i x}=\sqrt{\frac{\left|\int_{\Omega_{e}} \boldsymbol{\epsilon}\left(\boldsymbol{u}^{\text {bar } / \text { mix }}-\boldsymbol{u}^{\boldsymbol{v o l}}\right): \boldsymbol{E}: \boldsymbol{\epsilon}\left(\boldsymbol{u}^{\text {bar } / \text { mix }}-\boldsymbol{u}^{\boldsymbol{v o l}}\right)\right|}{\left|\int_{\Omega_{e}} \boldsymbol{\epsilon}\left(\boldsymbol{u}^{\boldsymbol{v o l}}\right): \boldsymbol{E}: \boldsymbol{\epsilon}\left(\boldsymbol{u}^{\boldsymbol{v o l}}\right)\right|}}
$$

$\eta_{e}^{b a r / m i x}$ is computed on the reference mesh, by projecting the stress and strain fields obtained with the bar and mixed methods. It must be noticed that this quantity does not really make sense to compare results computed on different meshes, since the stress and strain field are discontinuous at the boundaries between the finite elements. However for this test the meshes are exactly the same in the zone of interest (cf. figure 11), ensuring the relevance of (14) in this zone. The obtained errors are plotted in figure 15.

The most interesting observation is that the error in the zone of interest for the mixed method remains low (under 1.0\%). For the bar method, it is obviously high around the truss elements, but it can also reach about $5.0 \%$ even near the center of the beam. Outside of the zone of interest, the error is lower in the center of the beam for the mixed method even if conclusions must be considered with care since the meshes are not the same; in particular, some numerical artefacts can be observed. In both cases, the errors are high around the reinforcements, which was to be expected since (14) is not computed with the same Hooke tensor, depending on how the reinforcement is represented. Promising conclusions can be drawn from this example: in the zone of interest, the results obtained with the mixed method and the volumic method are very close to each other. The importance of using a volumic representation of the reinforcement (at least in the zone of interest) compared to a $1 \mathrm{D}$ representation was also emphasized. As expected, in the rest of the structure, the results are not as accurate as results that would be obtained with a fine mesh in the whole structure, however it seems that some improvement can be observed compared to the bar method.

\subsubsection{Bending of a 3D post}

This example illustrates how the mixed method can handle many reinforcements with complex geometries. The mesh of the post, the 1D mesh and the approximated geometry of the reinforcements are shown in figure 16. It is reminded that the only necessary information for the representation of the reinforcements is a 1D mesh and their radius, which is particularly interesting in this test case with many reinforcements and complex paths. Indeed, representing several 3D reinforcements which may be tangent to each other as shown in figure $16 \mathrm{~d}$. with a conforming mesh is very laborious. The lower surface at the base of the post is clamped, whereas the top of the post is subjected 

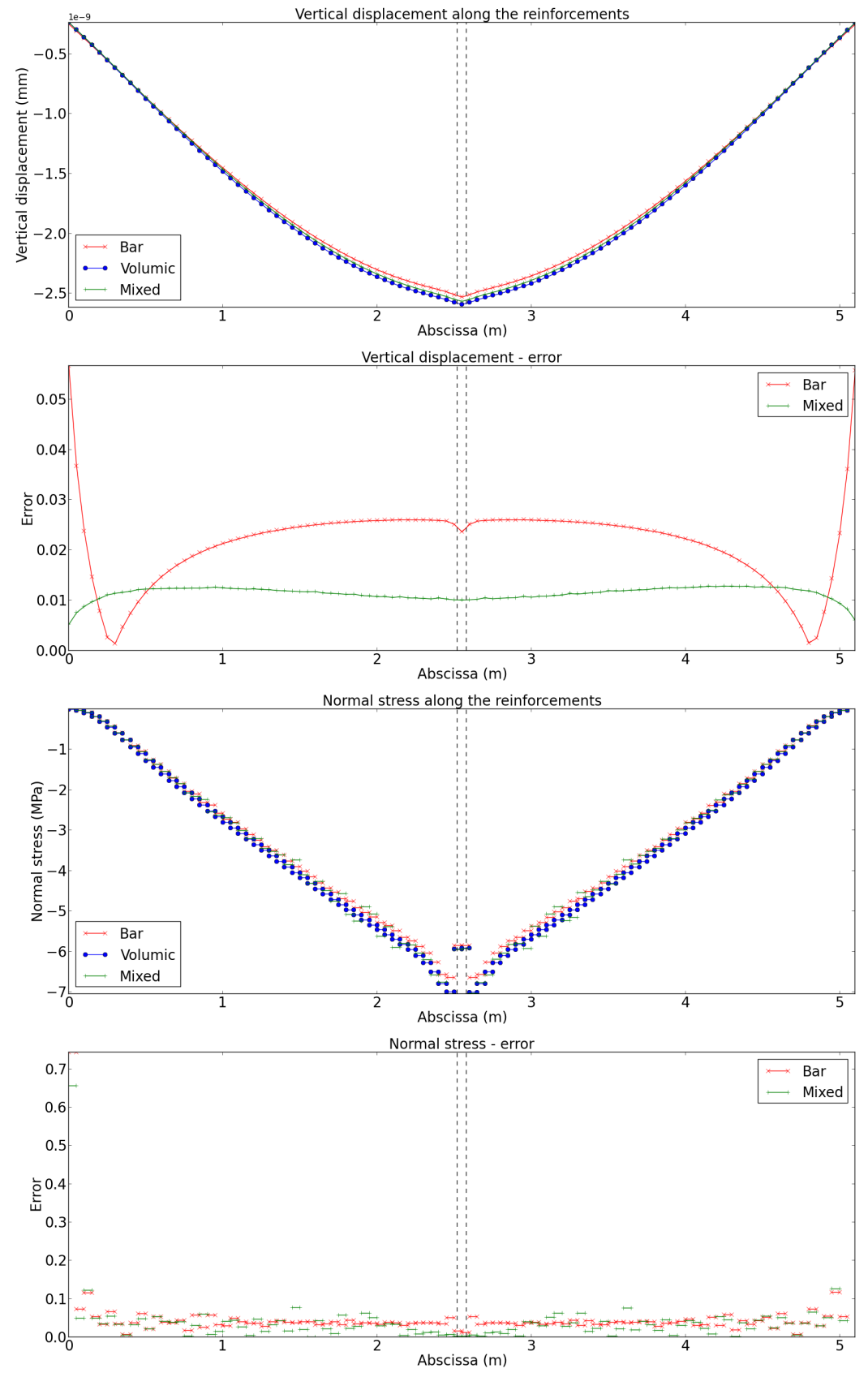

Figure 14: Displacements, normal stresses and errors in the upper reinforcement. The black dashed lines locate the limits of the zone of interest 


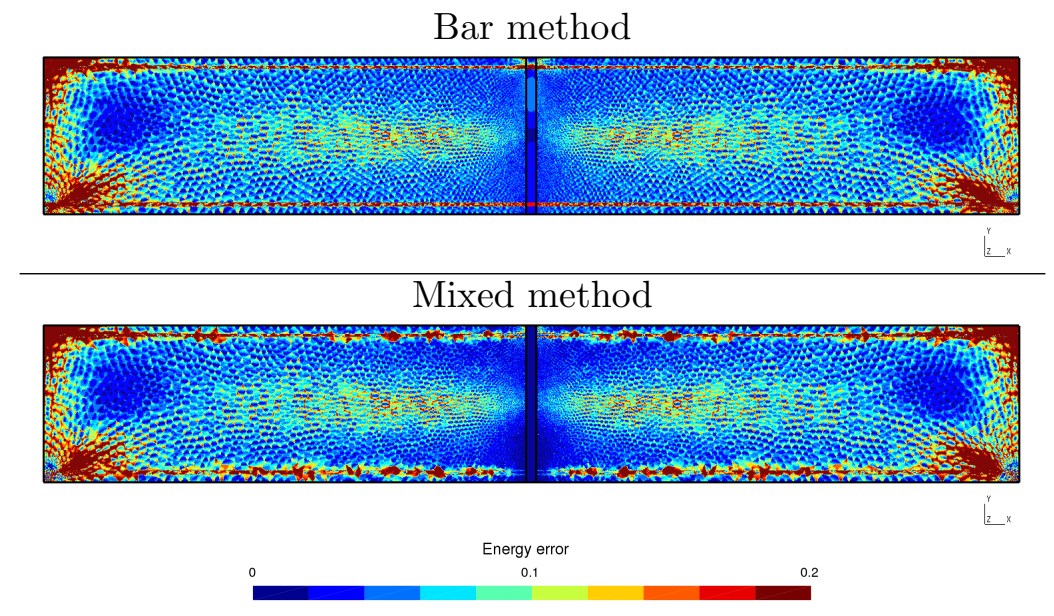

Figure 15: Energy errors. The maximum value was set to 0.2 for visibility: the red zones correspond to error greater than 0.2 .

to a shearing load. The zone of interest is supposed to be at the base of the beam, where the stress is expected to be the highest. To simplify the computations, only the reinforcements crossing the zone of interest are modeled using the mixed method (that is to say, the vertical reinforcements and two horizontal frames). The other reinforcements are modeled by 1D truss elements. The results of this computation are still referred as the "mixed" method (even if it is not true for all the reinforcements). The computational cost for a volumic representation of the reinforcements would be too high, therefore there is no reference computation for this example. Von Mises stresses in the zone of interest, in several cross-sections of the post, are shown in figure 17. Far away from the reinforcements, no significant differences can be observed between the bar and the mixed method. However, with the mixed method, a slight decrease of the Von Mises stress can be observed around the reinforcements boundaries (green dashed frames in figure 17).

\subsection{Plastic test cases}

In this section the mixed method is illustrated on non-linear examples. The matrix is made of an elastoplastic material with isotropic hardening. To avoid spurious appearance of plasticity around the part of the reinforcements which are only partially represented by the level-set, the plasticity is restrained to the zone of interest whereas the rest of the structure is assumed linear elastic. Usually the reinforcements are stronger than the surrounding matrix and yield for much higher loading, in some applications they do not yield at all; therefore they will be considered linear elastic in what follows. 


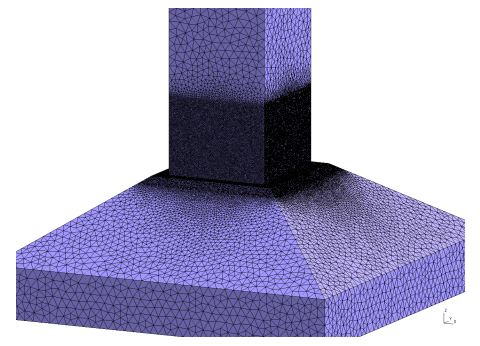

a.

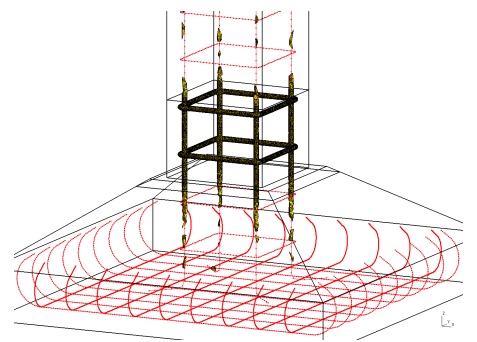

c.

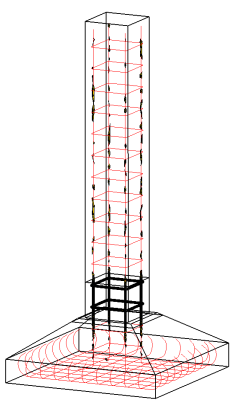

b.

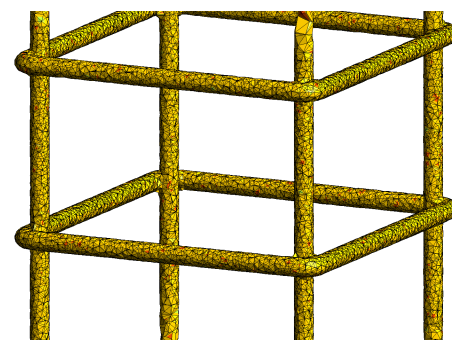

d.

Figure 16: a. 3D mesh of the post. b. Overview of the 1D mesh and of the approximated geometry of the reinforcements. The red lines correspond to the 1D mesh of the reinforcements. c. Zoom around the zone of interest. d. Zoom around the zone of interest, close to the 3D part of the reinforcements. 


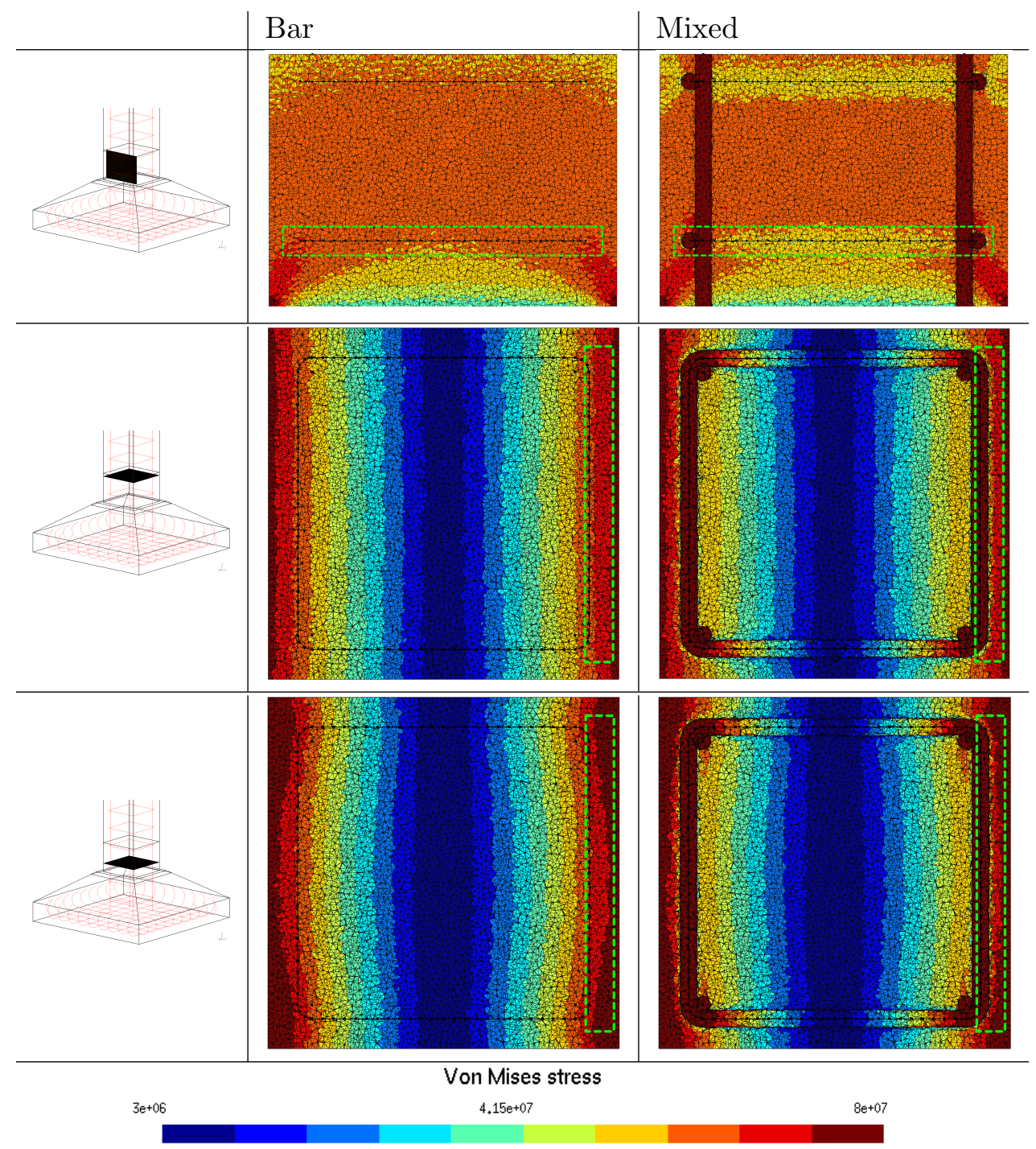

Figure 17: Von Mises stress in several cross-section views for the bending of the 3D post. 

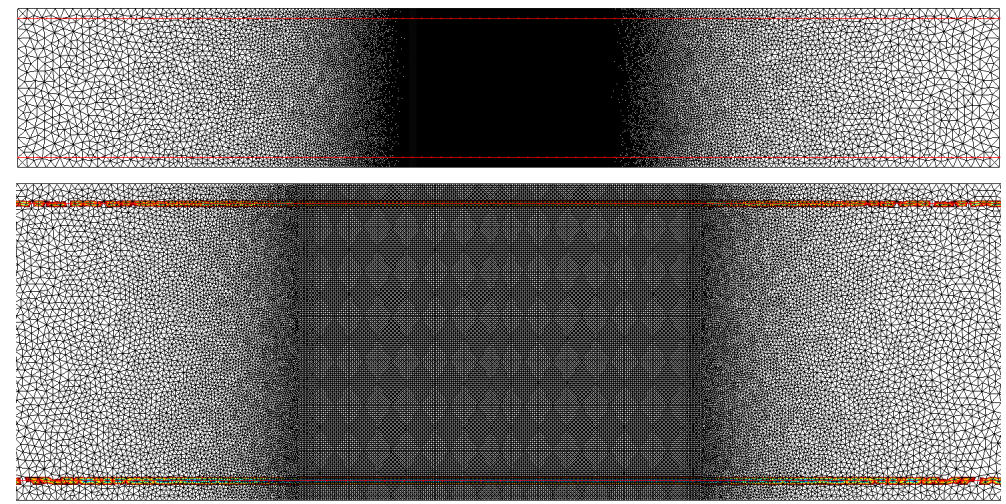

Figure 18: Top: Mesh used for the three points bending test. Bottom: geometry of the reinforcements approximated with level-set functions.

\subsubsection{Three points bending 2D plastic}

The test case of section 5.1.1 is considered with an elastoplastic matrix. The width of the zone of interest is $D=0.3 \mathrm{~m}$. The element size in the zone of interest is 0.005 $\mathrm{m}$. Corresponding computation meshes are shown in figure 18. The geometry of the reinforcements approximated with level-set functions on both meshes is represented as well. The plastic strain at the end of each computation for the different meshes and methods are given in figure 19.

In each computation, the plastic strain starts to develop under the loading zone, then at the bottom of the beam. However, with the bar method, it tends to reach the middle of the beam, whereas for the volumic method, the plasticity is confined around the reinforcements. The volumic method and the volumic part of the reinforcements in the mixed method introduce some heterogeneities, and therefore a stress field different from the bar method, leading to these differences in the results. With the mixed method, the plastic strain in the zone of interest is similar to the one obtained with the volumic method.

\subsubsection{D Four points bending test}

The next example is a $3 \mathrm{D}$ beam subjected to 4 points bending, with the following dimensions: length $L=5 \mathrm{~m}$, height $h=1 \mathrm{~m}$, thickness $t=0.5 \mathrm{~m}$. It is reinforced with four reinforcements (cf. figure 20) of radius $r=\frac{2}{9} t$, placed at a distance $c_{v}=0.25 \mathrm{~m}$ from the upper and lower faces of the beam, and $c_{h}=0.125 \mathrm{~m}$ from the lateral faces. The distance between the middles of the two loading zones is $b=0.75 \mathrm{~m}$ and the width of the zone of interest is $D=1 \mathrm{~m}$.

Using symmetry conditions, only a quarter of the beam is modeled; the displacement along the $x$-axis of the horizontal face located at $x=0$ is blocked, as well as the displacement along the $z$-axis of the horizontal face located at $z=L / 2$. The element 

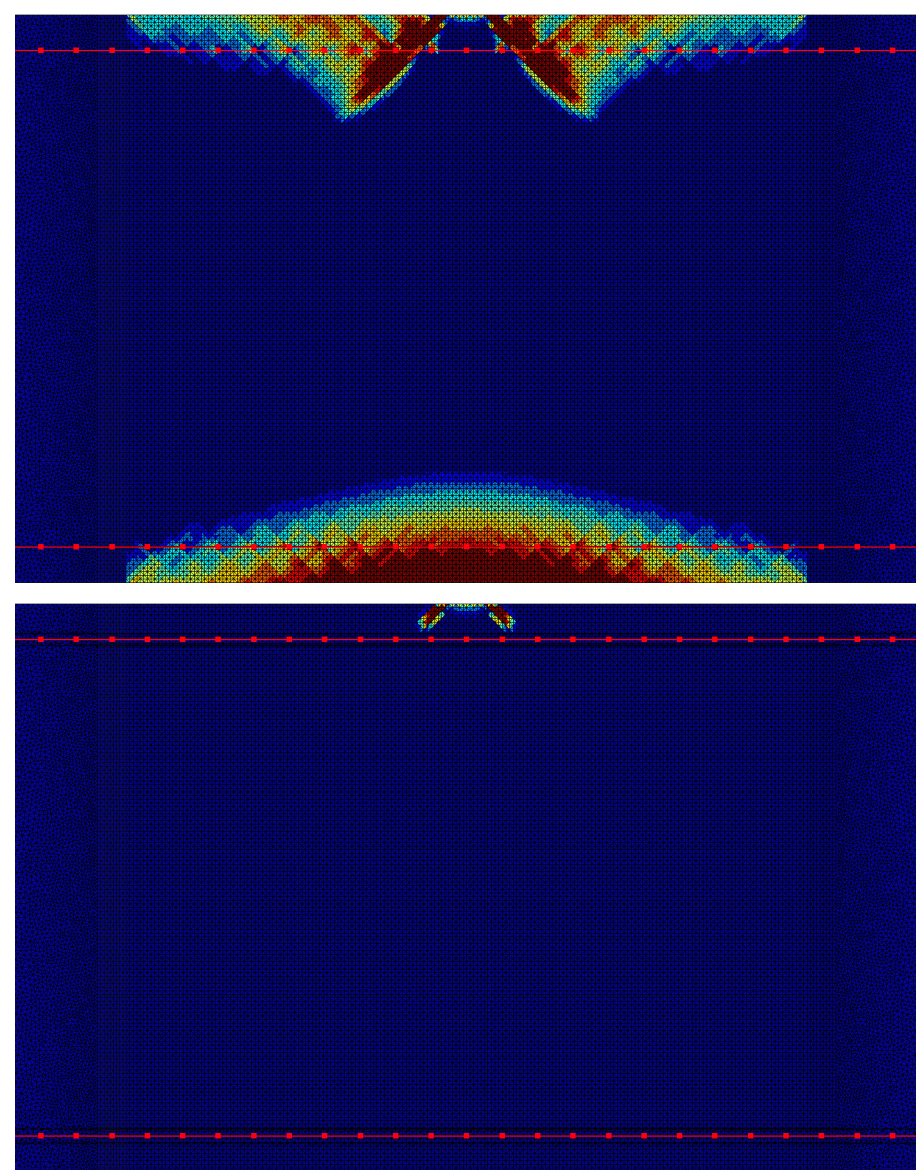

$4 \%$

Plastic strain

5e-05

Figure 19: Plastic strain at the end of the computation. Top: Bar method. Middle: Mixed method. Bottom: Volumic method. 

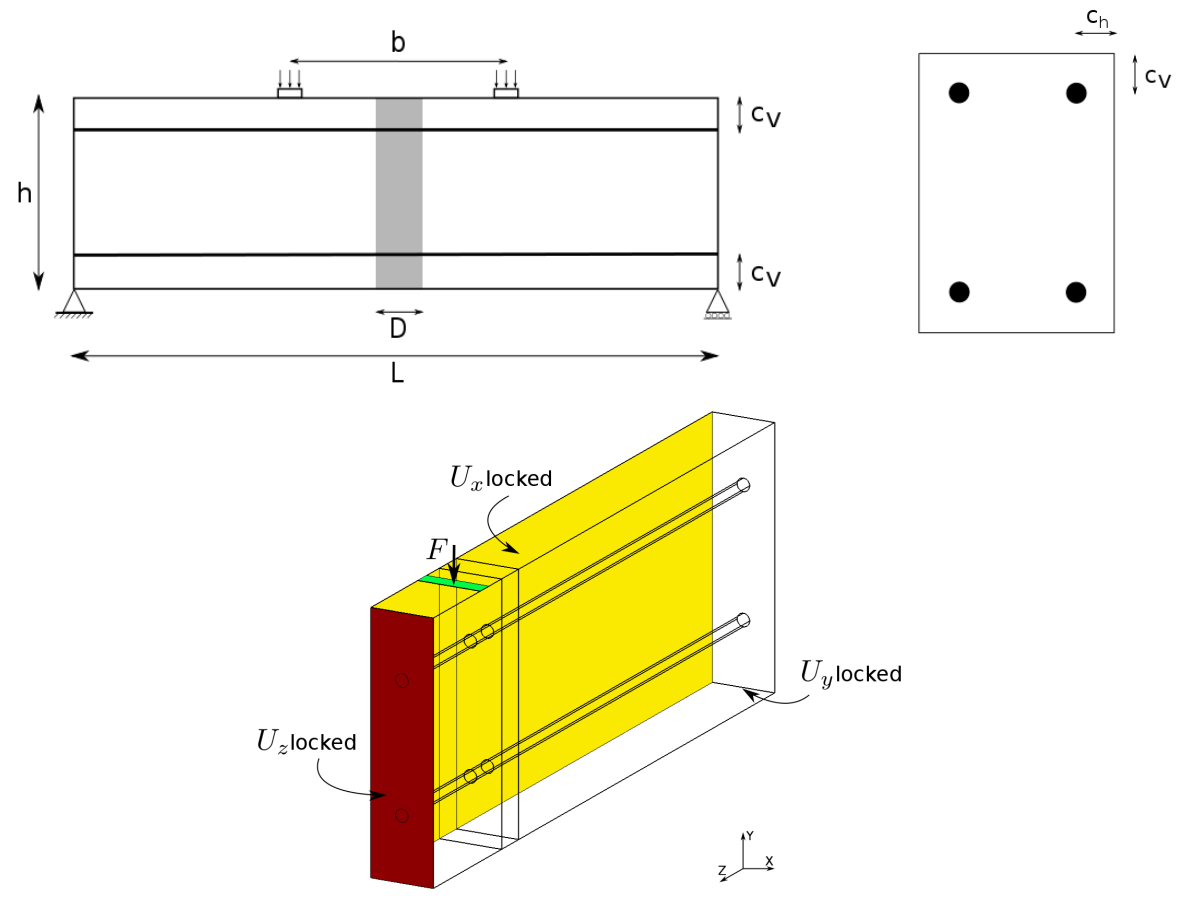

Figure 20: Geometry of the four points bending test and boundary conditions

size is $0.1 \mathrm{~m}$, except in the zone of interest where it was reduced to $0.05 \mathrm{~m}$ to better capture the non-linear effects. Note that this size is too large to represent correctly the reinforcements, but in order to decrease the computing times, the mesh was refined only around the reinforcements with a size of $0.005 \mathrm{~m}$ (cf. figure 21). For the computation mesh, this local refinement is concentrated in the zone of interest.

The geometry approximated with the level-set function is represented in figure 22 . The plastic strains in the zone of interest in different cross-sections of the beam and in the reinforcements plane, are given in figure 23. One can observe that when using the bar method, the bar elements tend to concentrate the plastic strain, near the nodes of the 1D mesh. This phenomenon was not observed in the example of the section 5.2.1. Indeed, in two dimensions finite elements with a size with the same order of magnitude than the reinforcements radius were enough to correctly represent them. In three dimensions, the curvature due to the cylindrical geometry of the reinforcements requires smaller elements (in this example the ratio between the elements size around the reinforcements and their radius is around $\frac{1}{20}$ ). This leads to stress concentrations around the 1D bars. Here again, the absence of heterogeneity leads to a plastic strain which is higher with the bar method, even far from the reinforcements. However, the results with the mixed method are almost the same as the ones obtained with the volumic method, which were the desired results.

Finally, to quantify the gain in CPU times, a sequential elastic computation was 

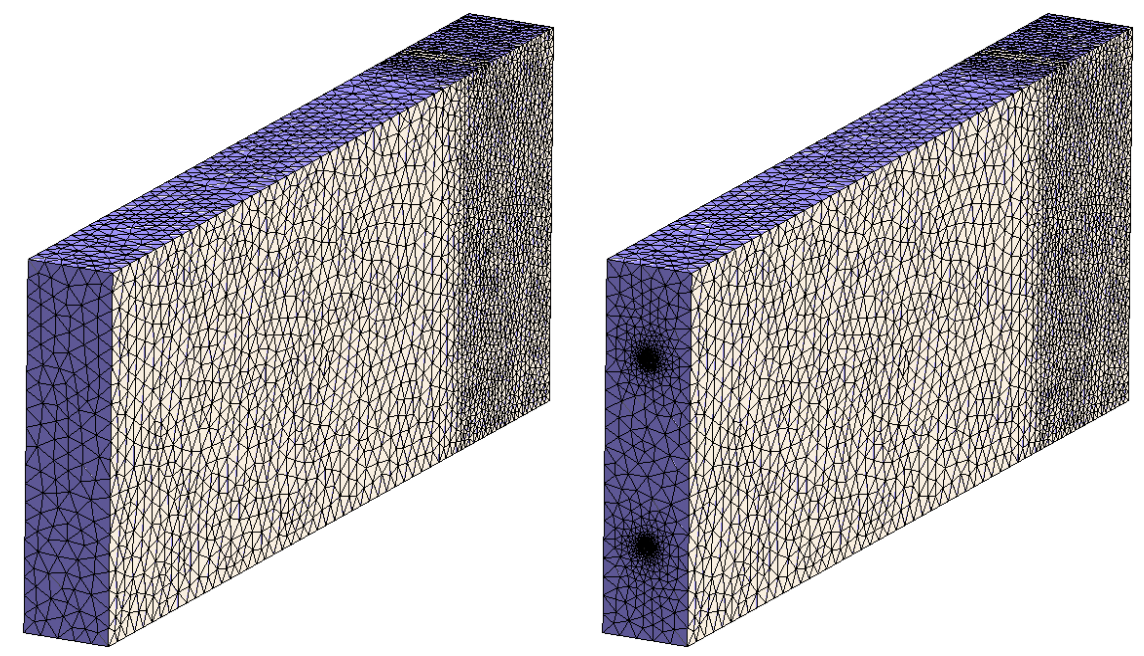

Figure 21: Meshes used for the four points bending test. Left: mesh used to test the bar and mixed methods. Right: mesh used to test the volumic method.

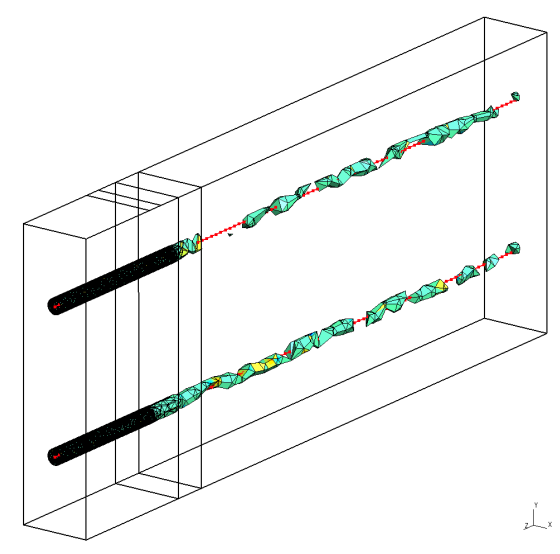

Figure 22: Approximation of the reinforcements in the four points bending test, using the mixed method

performed with each of the three methods. The number of degrees of freedom and the duration of different steps of the computation are given in table 2. The "Pre-treatment" step includes the definition of the X-FEM enrichment and the computation of coefficient $\alpha_{e}$.

The additional cost of the mixed method is quite low compared to the bar method, which is interesting considering that in the zone of interest, the obtained results are the same than the one obtained with the volumic method. 


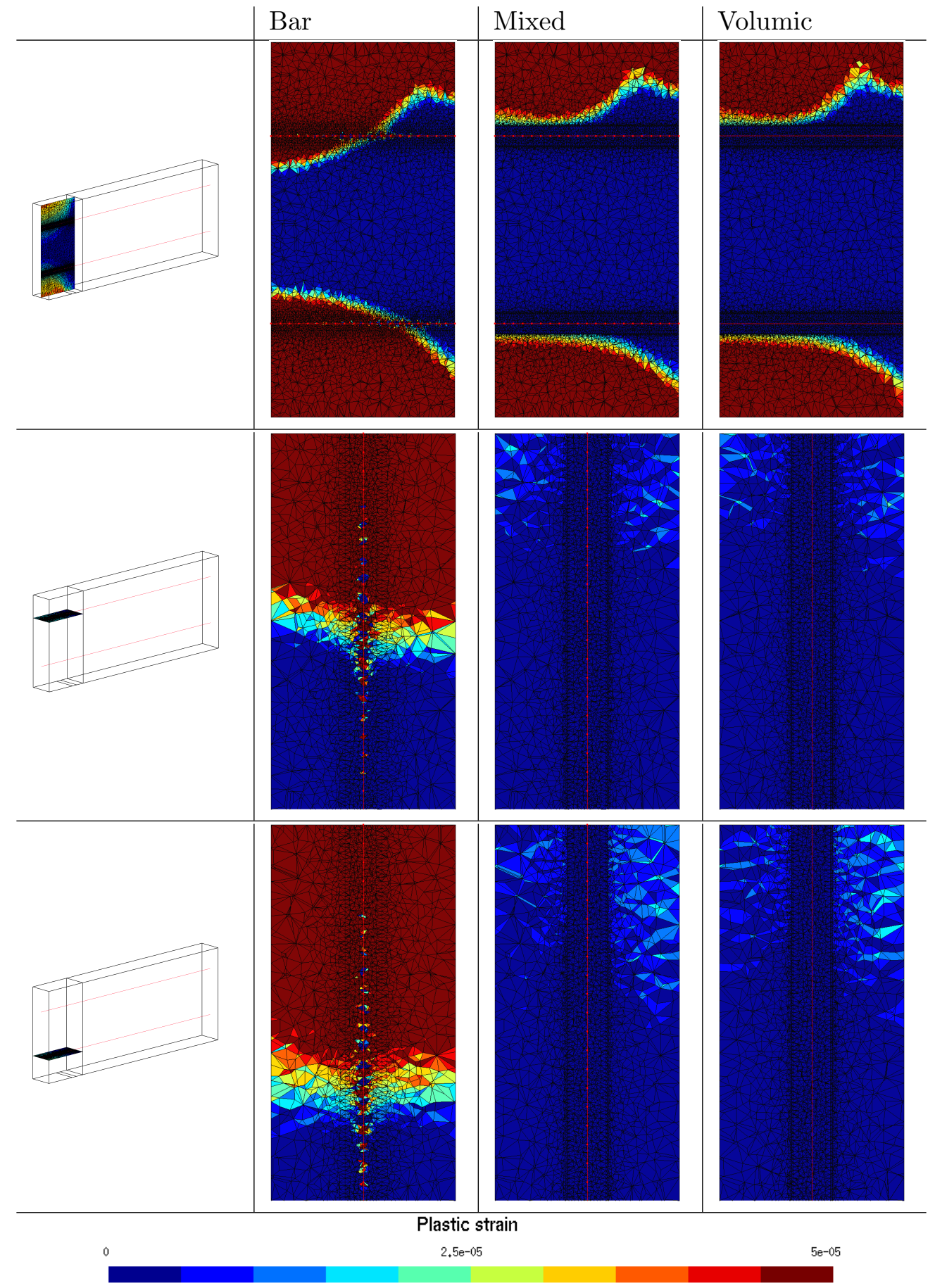

Figure 23: Plastic strain at the end of the computation in several cross-section views. 


\begin{tabular}{|l|l|l|l|l|}
\hline & $\begin{array}{l}\text { Number of de- } \\
\text { grees of freedom }\end{array}$ & $\begin{array}{l}\text { Pre- } \\
\text { treatment }\end{array}$ & Assembling & Solving \\
\hline Bar & 117909 & $2.68 \mathrm{~s}$ & $14.63 \mathrm{~s}$ & $11.06 \mathrm{~s}$ \\
\hline Mixed & 155190 & $36.96 \mathrm{~s}$ & $141.90 \mathrm{~s}$ & $36.96 \mathrm{~s}$ \\
\hline Volumic & 482677 & $142.01 \mathrm{~s}$ & $262.57 \mathrm{~s}$ & $50.97 \mathrm{~s}$ \\
\hline
\end{tabular}

Table 2: Number of degrees of freedom and computing times.

\subsubsection{Punching of a 3D slab}

The last example is a $3 \mathrm{D}$ slab. It is reinforced with two layers of perpendicularly and regularly spaced reinforcements of radius $0.015 \mathrm{~m}$. Each layer is made of 21 reinforcements in the $x$-axis direction and 21 reinforcements in the $y$-axis direction (for a total of 84 reinforcements). The upper layer of reinforcement is located $0.032 \mathrm{~m}$ from the upper boundary of the slab, and the lower layer is located $0.04 \mathrm{~m}$ from the lower boundary of the slab (distances measured from the center-line of the reinforcements). The dimensions of the slab are $3 \times 3 \times 0.25 \mathrm{~m}$ (cf. figure 24 ). The zone of interest is a parallelepiped with dimensions $0.3 \times 0.3 \times 0.25 \mathrm{~m}$ located at the middle of the slab, under the loading zone. The element size is $0.01 \mathrm{~m}$ in the zone of interest, and $0.15 \mathrm{~m}$ in the rest of the structure.

To decrease the computing time due to the treatment of the level-set and enrichment, the reinforcements crossing the zone of interest are modeled using the mixed method as described in section 4 (that is to say, 3 reinforcements by layer, in each direction), whereas the others are represented using only $1 \mathrm{D}$ bar elements. The geometry of these reinforcements approximated by X-FEM is shown in figure 25. For this test case, the number of degrees of freedom would be too high for a volumic representation, so there will be no reference computation. The lateral sides of the slab are clamped, and a vertical loading is applied at the center, on a square surface of dimensions $0.15 \times 0.15 \mathrm{~m}$.

The plastic strain in different planes (two horizontal planes crossing the upper and lower layer of reinforcements, and one vertical plane with a normal oriented along the $x$-axis, crossing the slab at $x=1.5$ ) is shown in figure 26 for the bar and mixed methods.

In this case, the element size and the reinforcements radius are close to each other, which explains why plastic strain concentration is not observed around the reinforcements for the bar method. However, differences can still be observed between the results obtained with the mixed and bar methods, showing the importance between a volumic and a $1 \mathrm{D}$ representation of the reinforcements.

\subsection{Toward a coupling with mesh adaptation methods}

The potential of the proposed approach is finally illustrated on a last example. Indeed, the coupling of this mixed dimensional model with mesh adaptation strategies is very appealing, as the scale of representation of the reinforcements changes dynamically with element size. Consider the three point bending test presented in section 5.1 in which 


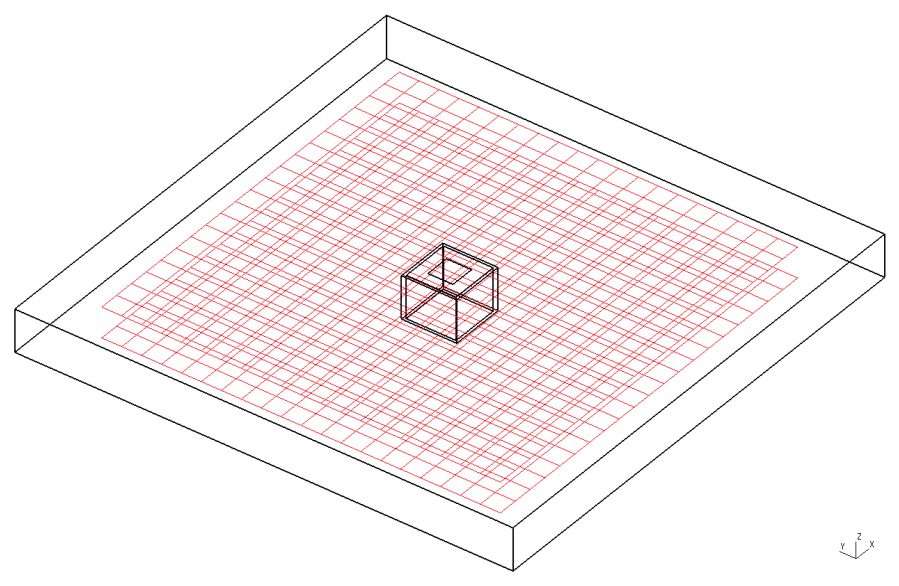

Figure 24: Geometry of the slab test

damaged zones are introduced thanks to the Thick Level Set (TLS) method [19]. The TLS approach is a method to regularize damage models, which allows a natural transition to discontinuous failure, relying on several key elements:

- A representation of the damage variable $d$ through an auxiliary field $\phi$, which is a level-set function. $d$ is written as a known function (parameter of the model) $d(\phi)$, usually called the damage profile

- A characteristic length $\ell_{c}$, corresponding to the regularization length as in most of the non-local damage models [17]

- An enrichment with the ramped Heaviside function of the iso- $\ell_{c}$ of $\phi$ which corresponds to the position of the cracks, to introduce discontinuities of the displacement field [20]

Note that no damage evolution is considered here: the damage zone is fixed (and frozen) at a given state of $\phi$ and the mechanical fields arising from this state are computed. $\phi$ is chosen in order to avoid fully damaged zone (corresponding to $d=1$ ), which would involve the treatment of the coupling between the enrichment representing the material interface (see section 2.1) and the ramped Heaviside enrichment (which will be the topic of further investigations). The quadratic damage profile proposed by [21] is used. Adapted meshes (see figure 27) are considered in order to track the damaged zone during its evolution and show the influence of this refinement on the representation of the mechanical fields. The resulting axial strain fields $\epsilon_{x x}$ along the axis of the beam are given, both for a standard 1D representation of the reinforcements and for the proposed mixed-dimensional method. Comparing those fields for different damaged states highlights the benefits of the proposed approach over classical bar models: mechanical fields are improved in the damaged area (i.e. where the accuracy of the model is of prime importance), especially around the reinforcements. The switch between bar and volumic 


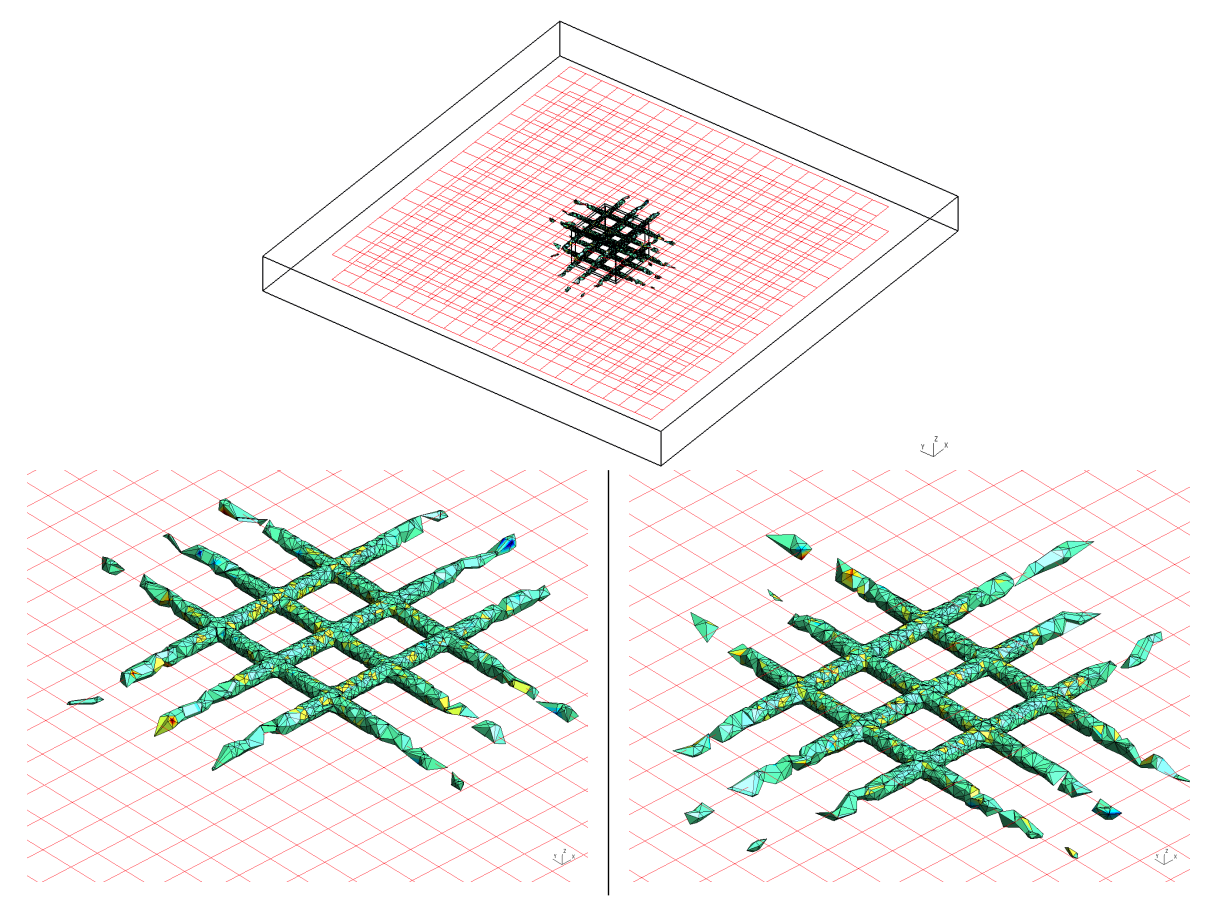

Figure 25: Geometry of the reinforcements approximated by level-set functions

models is handled automatically during the evolution of the damaged zone. Note also that, like in the previous examples, the length of the bar elements has to be carefully chosen as it can lead to stress concentration spots when this size is too large with respect to the elements.

\section{Conclusion}

A new approach to study reinforced structures is proposed, based on the idea that when performing a finite element analysis, it is most of the time possible to identify zones of interest. Fine meshes are used in these zones to get accurate results, whereas coarser elements are used in the rest of the structure to decrease computation times. In this framework, X-FEM is used to model the reinforcements, simplifying the meshing process. $\mathrm{X}$-FEM alone would give inaccurate results, because the geometry of the reinforcements would not be correctly represented in the coarse zone. The proposed solution consists in assembling simultaneously 1D truss elements, multiplying their elementary stiffness matrix by a coefficient depending on the local accuracy of the representation of the reinforcements by the level-set functions. This approach was validated through several examples, where the same results were obtained in the zone of interest, whether this approach or a full volumic representation of the reinforcements on a fine mesh is used.

The proposed method allows to combine a volumic and a lineic representation of the 


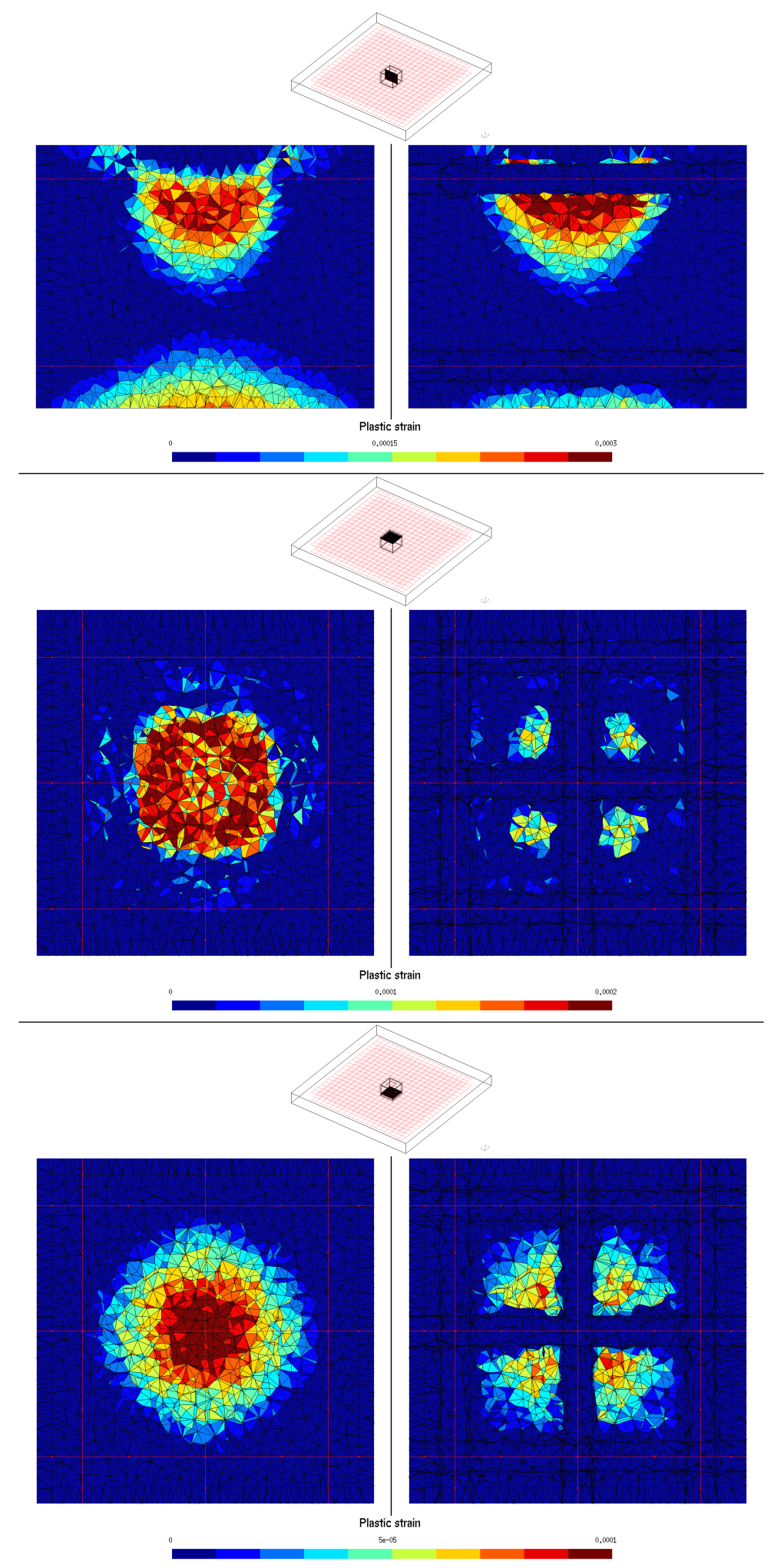

Figure 26: Plastic strain at the end of the computation in several cross-section views. 


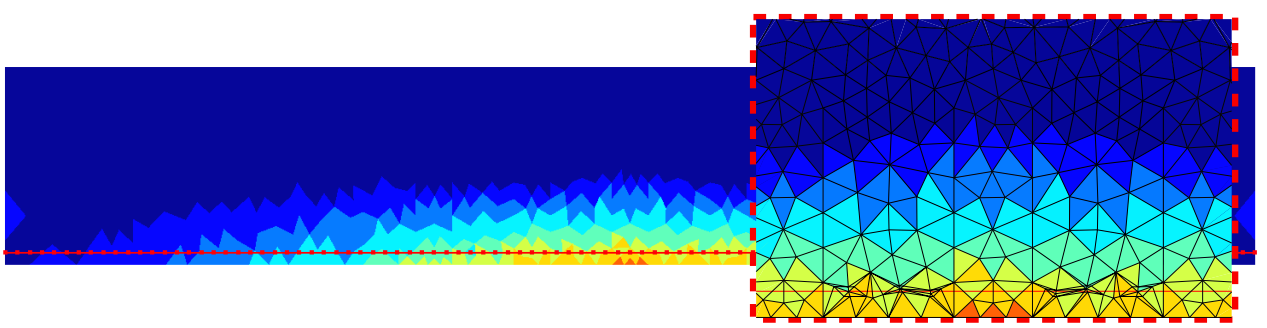

a.

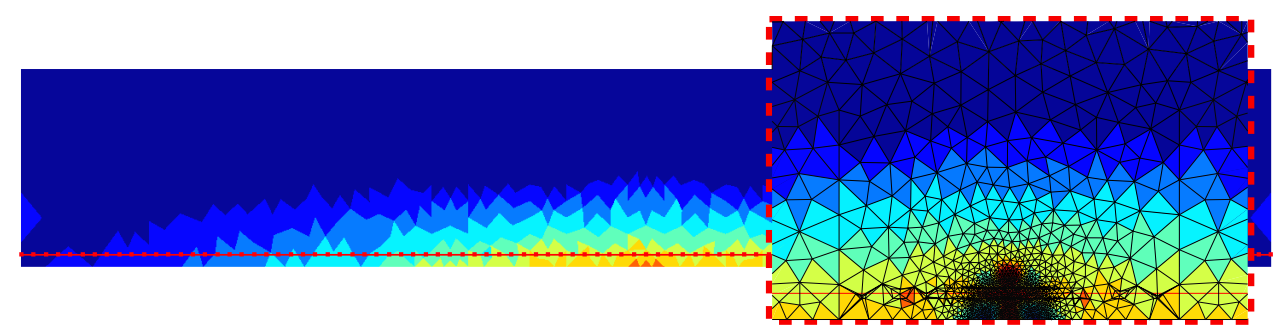

b.

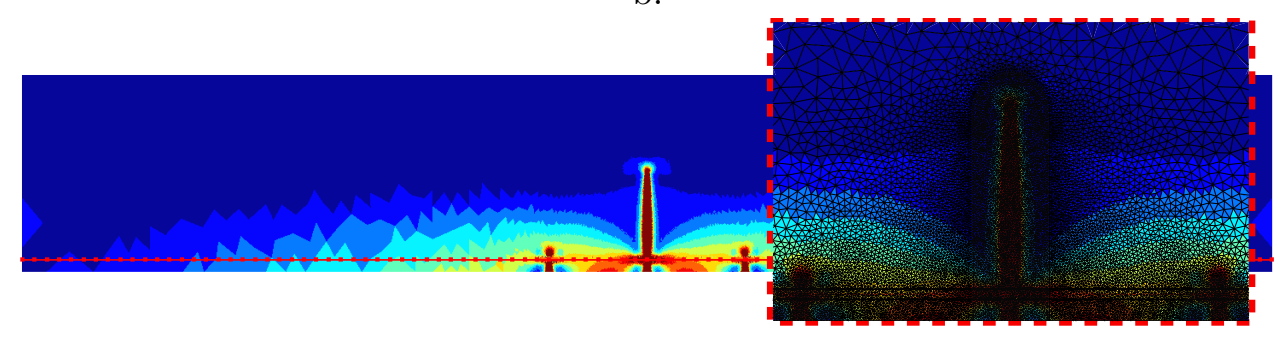

c.

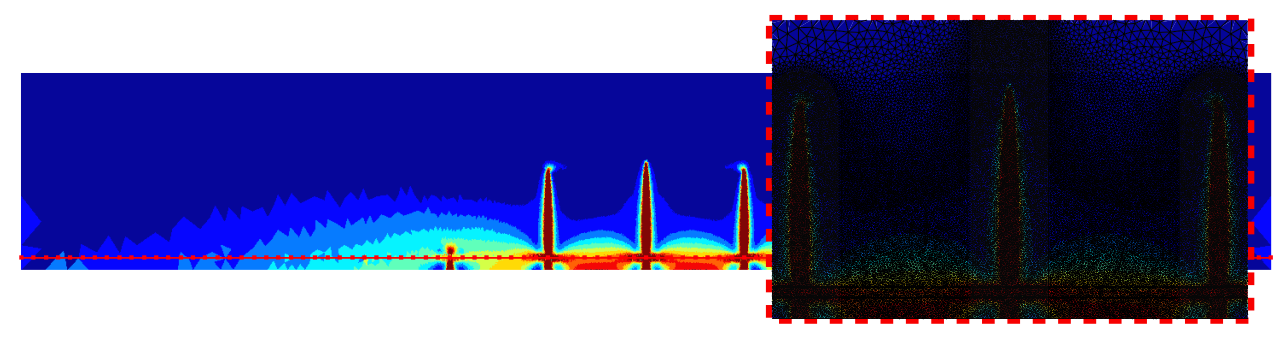

d.

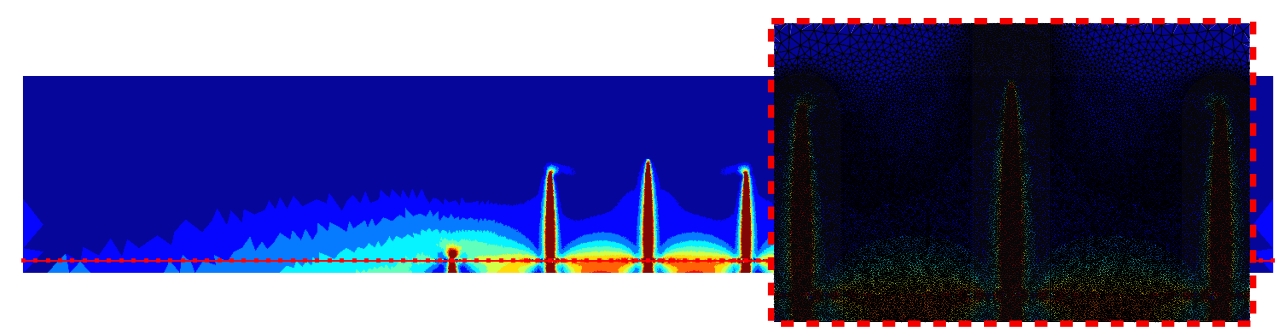

e.

eps_xx

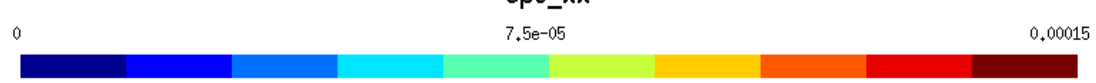

Figure 27: Axial strain $\epsilon_{x x}$ for different "remeshing" steps. From a. to d. : mixed method. e. bar method at the step $_{32}$ corresponding to d. 
reinforcements in the same computation. In the zone of interest, where the meshes are fine, a 3D representation of the reinforcements guarantees accurate results. In the rest of the structures, the use of coarser meshes associated with lineic elements decreases the computing times. The transition between the $3 \mathrm{D}$ and the $1 \mathrm{D}$ representation is such that the results in the zone of interest are almost identical to the results that would be obtained with a full volumic representation. Besides, several other advantages can be underlined:

- It is easy to implement, provided that one has a basic X-FEM library (enrichment functions and level-set, as well a element cutting for numerical integration). It can also be noticed that compared to most of the approach coupling $1 \mathrm{D} / 2 \mathrm{D}$ model to $3 \mathrm{D}$ model, it is not necessary to know exactly the interface between the $1 \mathrm{D}$ and the 3D zones; here both 1D and 3D models coexists, the transition between both is insured by the coefficients $\alpha_{e}$.

- The computational costs remain low: the computation of the coefficients $\alpha_{e}$ needs to be done only once, at the beginning of the analysis, therefore the computing costs related to this step are low, even in the case of a non linear analysis (unless some remeshing is involved).

- It is simple to use. One just needs to know the zones of interest, which is a common issue when performing a finite element analysis, and the 1D path of the reinforcements. The 3D part of the reinforcements is represented implicitly by $\mathrm{X}$ FEM, simplifying the meshing process, whereas the 1D mesh is independent from the volumic mesh.

It must be emphasized that the efficiency of the proposed approach is strongly dependent on the common sense of the user. For instance, one should should ensure that the mesh is fine enough in the zones of interest, and should not have a transition between $1 \mathrm{D}$ and 3D representation in these zones (as a reminder, the coefficients $\alpha_{e}$ corrects the global stiffness in the areas where X-FEM fails to represent the reinforcements, but does not provide accurate local results in these zones). As the geometry approximated by level-sets is piecewise linear 2.2, the density of the mesh in the zones of interest should be the same as the one that should be used to represent the reinforcements with a classical 3D mesh (that is to say, with nodes coinciding with the material interface). At least one order of magnitude between the reinforcement radius and the size of the elements in the fine zones seems to be a minimum in 3D.

This approach was developed considering perfect bond between the reinforcements and the surrounding matrix, however it is often not the case, as in reinforced concrete for instance where the steel-concrete interface is not perfect. If the reinforcements are represented using X-FEM, an approach similar to what is done in [22] for cohesive crack growth could be considered. The Ridge enrichment function could be replaced by a Heaviside enrichment function, to get a discontinuous displacement at the interface but with "cohesive" forces representing the bond between the reinforcements and the matrix. For reinforcements represented using bar elements, it is possible to consider 
a slip between the bar nodes and the surrounding volumic elements, and to introduce bond forces as in [16]. Future work will focus on how to deal with bond slip in the zones where both X-FEM and bar representation are superimposed $\left(0<\alpha_{e}<1\right)$.

\section{Appendix}

In order to illustrate the interest of using the eXtended Finite Element Method, a convergence test is performed on the bidimensional problem of a circular inclusion in a rectangular plate similar to the one depicted on figure 1 . The error is defined by

$$
\eta=\sqrt{\frac{\left|\int_{\Omega} \epsilon\left(\boldsymbol{u}^{h}-\boldsymbol{u}^{e x}\right): E: \epsilon\left(\boldsymbol{u}^{h}-\boldsymbol{u}^{e x}\right)\right|}{\left|\int_{\Omega} \boldsymbol{\epsilon}\left(\boldsymbol{u}^{e x}\right): E: \epsilon\left(\boldsymbol{u}^{e x}\right)\right|}}
$$

where $\boldsymbol{u}^{\boldsymbol{e x}}$ is the exact solution and $\boldsymbol{u}^{\boldsymbol{h}}$ an approximate solution. $\boldsymbol{u}^{\boldsymbol{h}}$ is calculated with three different methods:

- The classical finite element method (FEM) with a mesh conforming to the material interface.

- A finite element computation with an arbitrary mesh, not conforming to the material interface. The numerical integration is performed using the procedure described in section 2.2 , i.e. with subcells but no enrichment.

- A computation with the same non-conforming mesh, using the X-FEM.

The error is plotted for the three methods on figure 28. It can be observed that the $\mathrm{X}$-FEM allows to recover a convergence rate which is close to the conforming FEM, whereas the non-conforming FEM gives a poor convergence rate. For a given element size, the non-conforming FEM is always the less accurate method.

In order to get information about the local accuracy of each method, the local error for each element of the mesh $e$ is defined:

$$
\eta_{e}=\sqrt{\frac{\left|\int_{\Omega_{e}} \boldsymbol{\epsilon}\left(\boldsymbol{u}^{h}-\boldsymbol{u}^{e x}\right): \boldsymbol{E}: \boldsymbol{\epsilon}\left(\boldsymbol{u}^{\boldsymbol{h}}-\boldsymbol{u}^{e x}\right)\right|}{\left|\int_{\Omega_{e}} \boldsymbol{\epsilon}\left(\boldsymbol{u}^{e x}\right): \boldsymbol{E}: \boldsymbol{\epsilon}\left(\boldsymbol{u}^{e x}\right)\right|}}
$$

The local error for each method, for a ratio $\frac{h}{r}=\frac{1}{4}$ where $h$ is the element size and $r$ the radius of the inclusion, is shown on figure 29. For the three methods, the local error is concentrated around the material interface. However, the error obtained with the $\mathrm{X}$-FEM is of the same order of magnitude than the error obtained with the conforming FEM, which is of course lower than the error obtained with the non-conforming FEM. Note that the chosen ratio $\frac{h}{4}=\frac{1}{r}$ is quite representative of a typical element size required to study a circular inclusion (good compromise between the approximation of the curvature of the geometry and a reasonable mesh refinement). One could have imagined that for a fine enough mesh, the error committed with the non-conforming FEM would be small, which is not the case here. This example shows that, for a practical element 


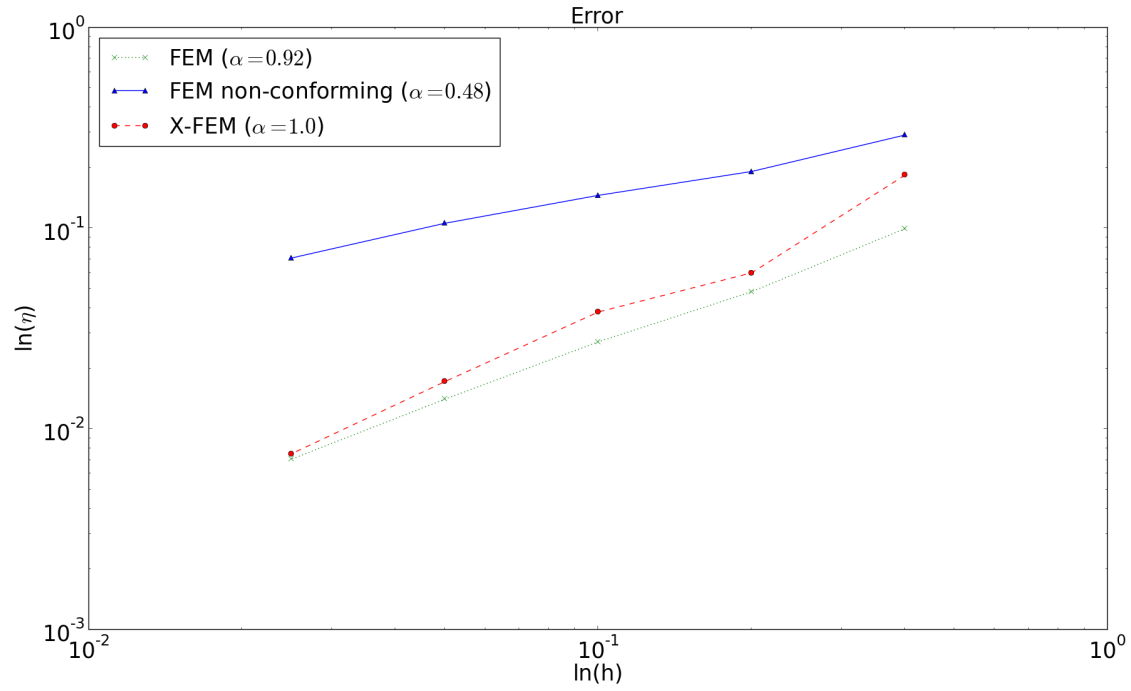

Figure 28: Convergence rates for the 2D inclusion problem.

size, the non-conforming FEM is not as accurate as the conforming FEM, which is not the case of the X-FEM.

\section{Acknowledgment}

The support of the ERC Grant XLS n0 291102 is gratefully acknowledged. 


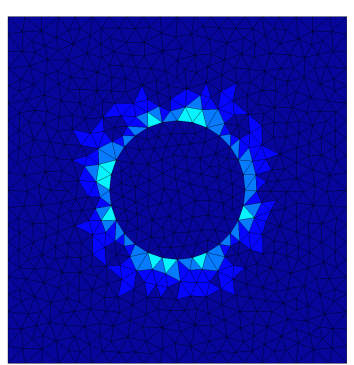

a.

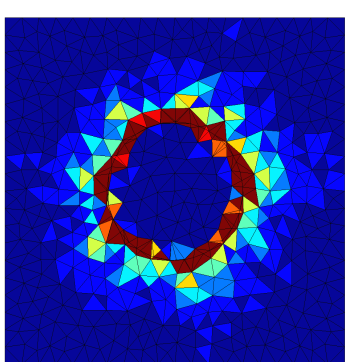

b.

Local error

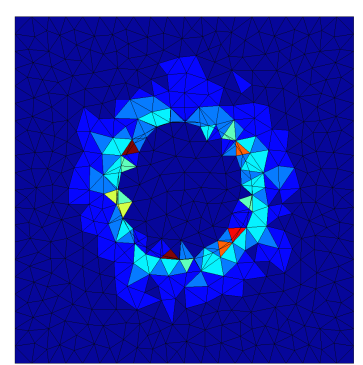

c.

0.005

Figure 29: Local error for the 2D inclusion problem for a ratio $\frac{h}{4}=\frac{1}{4}$. a. FEM. b. FEM non-conforming. c. X-FEM

\section{References}

\section{References}

[1] M. Suidan, W. C. Schnobrich, Finite Element Analysis of Reinforced Concrete, Journal of The Structural Division 99 (10) (1973) 2109-2122.

[2] M. David, J.-J. Marigo, C. Pideri, Homogenized interface model describing inhomogeneities located on a surface, Journal of Elasticity 109 (2) (2012) 153-187. doi:10.1007/s10659-012-9374-5.

[3] H. Hartl, L. Sparowitz, A. Elgamal, The 3D Computational Modeling of Reinforced and Prestressed Concrete Structures, in: 3rd International PhD Symposium in Civil Engineering, Vienna, Vol. 2, 2000, pp. 69-79.

[4] H. M. Gomes, A. M. Awruch, Some aspects on three-dimensional numerical modelling of reinforced concrete structures using the finite element method, Advances in engineering software 32 (4) (2001) 257-277. doi:10.1016/S0965-9978(00)00093-4.

[5] L. Jason, S. Ghavamian, A. Courtois, Truss vs solid modeling of tendons in prestressed concrete structures: Consequences on mechanical capacity of a Representative Structural Volume, Engineering Structures 32 (6) (2010) 1779-1790. doi:10.1016/j.engstruct.2010.02.029.

[6] D. J. Monaghan, I. W. Doherty, D. Mc Court, C. G. Armstrong, Coupling 1D Beams to 3D Bodies, in: 7th International Meshing Roundtable, Sandia National Laboratories, 1998, pp. 285-293.

[7] R. W. Mccune, C. G. Armstrong, D. J. Robinson, Mixed-dimensional coupling in finite element models, International Journal for Numerical Methods in Engineering 49 (6) (2000) 725-750. 
[8] V. P. Nguyen, P. Kerfriden, S. Claus, Nitsche's method method for mixed dimensional analysis: conforming and non-conforming continuum-beam and continuumplate coupling (2013) 0-1.

[9] P. Hansbo, Nitsche's method for interface problems in computational mechanics, GAMM-Mitteilungen 28 (2) (2005) 183-206. doi:10.1002/gamm.201490018.

[10] M. Sadek, I. Shahrour, A three dimensional embedded beam element for reinforced geomaterials, International Journal for Numerical and Analytical Methods in Geomechanics 28 (9) (2004) 931-946. doi:10.1002/nag.357.

[11] N. Sukumar, D. L. Chopp, N. Moës, T. Belytschko, Modeling holes and inclusions by level sets in the extended finite-element method, Computer Methods in Applied Mechanics and Engineering 190 (2001) 6183-6200.

[12] N. Moës, J. Dolbow, T. Belytschko, A finite element method for crack growth without remeshing, International Journal for Numerical Methods in Engineering 150 (February) (1999) 131-150.

[13] I. Babuška, The partition of unity method, International Journal for Numerical Methods in Engineering 40 (July 1995) (1997) 727-758.

[14] N. Moës, M. Cloirec, P. Cartraud, J.-F. Remacle, A computational approach to handle complex microstructure geometries, Computer Methods in Applied Mechanics and Engineering 192 (28) (2003) 3163-3177. doi:10.1016/S0045-7825(03)00346-3.

[15] T. Zohdi, M. Feucht, D. Gross, P. Wriggers, A description of macroscopic damage through microstructural relaxation, International Journal for Numerical Methods in Engineering 43 (3) (1998) 493-506. doi:10.1002/(SICI)10970207(19981015)43:3¡493::AID-NME461¿3.0.CO;2-N.

URL http://compmat.berkeley.edu/zohdipaper/4.pdf

[16] A. Casanova, L. Jason, L. Davenne, Bond slip model for the simulation of reinforced concrete structures, Engineering Structures 39 (2012) 66-78. doi:10.1016/j.engstruct.2012.02.007.

URL http://linkinghub.elsevier.com/retrieve/pii/S014102961200065X

[17] Z. P. Bažant, Instability, Ductility and Size-effect in Strain-Softening Concrete, Journal Of The Engineering Mechanics Division 102 (2) (1976) 331-343.

[18] J. C. Simo, T. J. Hughes, Computational Inelasticity, springer Edition, Berlin, 1998.

[19] N. Moës, C. Stolz, P. É. Bernard, N. Chevaugeon, A level set based model for damage growth : the thick level set approach, International Journal for Numerical Methods in Engineering 86 (3) (2011) 358-380.

[20] P. É. Bernard, N. Moës, N. Chevaugeon, Damage growth modeling using the Thick Level Set (TLS) approach: Efficient discretization for quasi-static loadings, 
Computer Methods in Applied Mechanics and Engineering 233-236 (2012) 11-27. doi:10.1016/j.cma.2012.02.020.

[21] A. Parrilla Gómez, N. Moës, C. Stolz, Comparison between Thick Level Set (TLS) and cohesive zone models, Advanced Modeling and Simulation in Engineering Sciences 2 (1) (2015) 1-22.

[22] N. Moës, T. Belytschko, Extended finite element method for cohesive crack growth, Engineering Fracture Mechanics 69 (7) (2002) 813-833. doi:10.1016/S00137944(01)00128-X. 